\title{
Incidencia de la política económica predominante sobre algunos parámetros de la seguridad alimentaria en EI Salvador*
}

\author{
Roberto Rublo
}

\section{Introducción}

La seguridad alimentaria de un país es uno de los aspectos más fundamentales de su desarrollo, especialmente en el caso de los países del llamado Tercer Mundo. Por un lado, una producción suficiente de alimentos y su distribución adecuada son elementos esenciales para el logro de un proceso de crecimiento/acumulación sostenido y equilibrado. En electo, la insuliciencia alimentaria puede afectar la tasa de salarios y en consecuencia la de acumulación, hace incurrir en fuertes importaciones que pesan sobre la balanza de pagos y sobre los "grados de dependencia", puede incidir negativamente sobre la importante industria alimenticia, etc. Asimismo, la inadecuada distribución alimentaria puede favorecer los ya marcados desequilibrios regionales y locales, asi como la inaccesibilidad a los productos básicos (o su mera insuficiencia) puede promover verdaderas "revuellas sociales" que dafian u obslaculizan la dinámica de las inversiones (ejemplos son las "revueltas" del pan, del trigo, del transporte, etc. habidas en Argelia, Túnez, Venezuela).

- La elaboración de este trabajo ha sido posible gracias al linanciamiento de la Auloridad Sueca para el Desarrollo Internacional (ASDI), a través del Instituto de Investigaciones Económicas y Sociales de la UCA (IIES-UCA). 
Por otro lado, la seguridad alimentaria es importante en términos de las posibilidades de salisfacer las necesidades básicas de las mayorias y de la elevación de la calidad de sus formas de vida. La situación alimenlaria y/o nutricional de un pueblo es esencial en la delerminación de su eslado de salud física ( $y$ por ende mental), en sus niveles de productividad, en sus "grados de creatividad", en los usos y/o distribución de los tiempos (no sólo el liempo dedicado al ocio, al trabajo, al esparcimiento, sino también al tipo de uso dado a cada uno de ellos), y en general para la posibilidad de mantener un organismo sano y "abierto a la emancipación del espíritu".

Dada la importancia de la seguridad alimentaria para el desarrollo de los pueblos, se hacen necesarios estudios que profundicen en sus caracteristicas y en los laclores que contribuyen a conligurarlas. En este sentido, el objelivo general del presente trabajo es analizar los electos que han tenido las políticas económicas predominantes en nuestro pais desde la década de los ochenta sobre la seguridad alimentaria de los salvadoreños.

Ahora bien, en razón de su imporlancia, el tema de la seguridad alimentaria ha sido objeto de variadas investigaciones. Entonces, ¿por qué volcarnos también sobre él?. En primer lugar, porque no son muchos los trabajos que relacionan la seguridad alimentaria con el entorno y la política macroeconómica. Esto afiade una importancia suplementaria, ya que en dicha relación se ponen en juego argumentos sobre la viabilidad y/o conveniencia de determinadas políticas económicas, más concretamente de las contenidas en los programas de estabilización económica y ajuste estructural. En segundo lugar, y esto es lo fundamental, porque la mayoria de investigaciones (al menos las consultadas) poseen un concepto limitado de la seguridad alimentaria (SA), que normalmente deja de lado importantes aspectos que creemos deben ser considerados dentro de la misma.

Por lanto, es menester pregunlarnos previamente ¿qué vamos entender por seguridad alimentaria? Comenzaremos respondiendo por la vía de las definiciones negativas, es decir, sefialando lo que a nuestro entender no es o no debe ser la SA. En lal sentido, la SA no se limita a que un pais disponga de una oferla suticiente de bienes alimenticios (como lo plantean los enfoques más clásicos), ni tampoco a que esa oferta sea accesible a la población (como lo plantean enfoques redistributivos más recientes). Podrán haber alimentos suficientes y accesibles a los bolsillos de las mayorias $y$, sin embargo, con ello no garanlizariamos la SA. Aunque la disponibilidad equilaliva sea un elemenlo importante de la SA, ésla va más allá de los criterios del crecimiento y la equidad. 
¿Qué es, entonces, para nosotros la SA?. Para responder (esla vez por la via de las definiciones afirmalivas), lomaremos como punto de partida dos grandes criterios que miden la imporlancia de analizar la seguridad alimenlaria. Nos relerimos a la posibilidad de contribuir a un proceso de crecimiento/acumulación sostenido y contribuir a un proceso de crecimiento/acumulación sostenido y equilibrado, y a la posibilidad de tavorecer la satisfacción de las necesidades básicas de las mayorias y mejorar la calidad de sus formas de vida.

Garanlizar un crecimiento de acumulación basado en la satisfacción de las necesidades básicas de las mayorías y en la mejora de la calidad de sus formas de vida tiene que ver con muchas cosas. Entre algunas de ellas podemos mencionar: una oferla suficiente de bienes y servicios, expansión robusla de la capacidad producliva, ciertos equilibros de las variables macroeconómicas, dinámica económica relativamente estable, distribución equitativa de los medios de producción y equidad en el acceso a los bienes y servicios producidos, capacidad de elaborar y ejecutar un proyecto nacional propio, buena calidad de los bienes y servicios, adecuada gestión del capital nalural (ecosistema) y del capital humano. alto nivel organizativo y democratización del proceso de loma de decisiones, etc.

Bajo tal contexto, la SA de una nación deberá medirse en términos semejantes. Un país "estará seguro", en lérminos alimentarios, en la medida que:

a) Disponga de una oferta suficiente de bienes (producidos nacionalmente o importados) y servicios alimentarios (redes de almacenamiento, canales de comercialización, sistemas de conservación y transporte, centros de distribución/venta, elc.).

b) Contenga una adecuada y robusla capacidad de producción de alimentos (que no lo hagan depender tanto de las imporlaciones y/ o que eslé en capacidad de responder a restricciones evenluales del mercado internacional).

c) Los alimenlos sean accesibles a la mayoria de la población.

d) Se asegure un abastecimienlo estable de alimenlos (en cantidàdes y precios)

e) Se garanlice una independencia alimentaria del exterior (lo cual no significa el autoabastecimienlo).

f) Las formas de crecer y acumular dentro del sistema alimentario sean ecológicamente viables (que no destruyan los equilibrios del ecosistema y aprovechen los benelicios que éslos ofrecen). 
g) Que se mejore la calidad de los alimenlos y de la dieta de las mayorías.

Asi, los parámetros en los que basaremos nuestras apreciaciones/ valoraciones sobre el estado de la SA en El Salvador serian los siguienles: ${ }^{1}$

\section{Suficiencia/disponibilidad}

Podemos hablar de suficiencia y/o disponibilidad alimentaria cuando "el sislema alimenlicio esté en capacidad de asegurar una oferta interna de alimentos (producción local e importaciones), cuyo volumen y composición sea suficiente para salisfacer adecuadamente las necesidades alimenticias de la población". Al respecto habria que hacer tres observaciones:

- La satisfacción se refiere no sólo a la demanda efectiva de la población, sino lambién a la demanda potencial. La SA tiene que ver no sólo con los que tienen capacidad de comprar alimenlos, sino con todos los ciudadanos.

- La disponibilidad de la oferla concierne sólo la destinada al consumo humano interno. Podrá haber una oferta abundanle, que leóricamente cubre la demanda potencial, pero si una alta proporción de la misma se destina al consumo animal, a la exporlación o al uso de la industria no alimentaria, tal abundancia podrá ser compatible con la inseguridad alimentaria.

- La suficiencia no sólo compete a la oferla de bienes alimenticios, sino también a la suliciencia de servicios vinculados al sistema alimenticio. Así, por ejemplo, las cuenlas nacionales podrán regisırar un volumen y composición de la olerta suliciente/disponible para la población en un momento determinado, pero la SA se vería aleclada si esa olerta posee grandes obstáculos para ser canalizada o comercializada, si la intraesInclura de almacenamiento y conservación reporta grandes pérdidas posicosecha, si la red de comunicaciones y/o la distribución geográfica de los centros de venta no eslá tan disponible a los compradores.

1. Los parámetros acá ulilizados toman como marco de referencia los indicadores sobre seguridad alimenlaria propueslos por la FAO. Hemos inlentado relormular y completar algunos de eslos y añadido uno más (el que alañe la calidad de los alimentos y la diversidad de la diela). Los parámelros se han tomados de Peitez, W., 1991. pp. 117-118). Las cilas que aparecen a continuación son tomadas de este Irabajo. 


\section{Equidad/accesibilidad}

Esle criterio de la SA "hace referencia a la necesidad de que toda la población, independientemente de su nivel de ingreso, tenga acceso a los alimentos necesarios para la reproducción, es decir, fuera de un marco de desnutrición". Como se notará, la accesibilidad a los alimentos en un régimen de mercado es inseparable de la equidad que debe exislir en la distribución de los ingresos.

\section{Estabilidad/confiabilidad}

Esto tiene que ver con "la capacidad de contar con mecanismos eficaces para neutralizar los eleclos que sobre el suministro y los precios puede tener un comportamiento inesperado de la producción nacional o de los precios de los alimentos imporlados". En la medida que los flujos de alimentos y niveles de precios de los mismos guarden cierta eslabilidad, el sislema alimentario tendrá mayor conliabilidad.

\section{Autonomia/vulnerabilidad}

Este parámetro apunta a que "el nivel de importaciones de alimenlos, insumos y bienes de capilal requeridos por el sistema alimentario para lograr la suficiencia sea tal que las flucluaciones del mercado alimentario mundial resulten manejables para la economia del país. Lo que se busca, por lo tanto, no es un estado de autarquia, sino la reducción de la vulnerabilidad externa". En este sentido, seguridad alimentaria no es equivalente al concepto de aulosuficiencia alimentaria.

\section{Sustentabilidad/viabilidad ecológica}

Con la suslenlabilidad, "lo que se busca no es una seguridad alimentaria de carácler lemporal, sino una de carácter permanenle. Para ello es necesario percatarse de que la suliciencia, la aulonomia y la estabilidad están siendo logradas sin comprometer la preservación de los recursos nalurales ni el control de los desequilibrios macroeconómicos". Ahora bien, no sólo se Irata de preservar los equilibrios del ecosistema. Un sistema alimentario será "más seguro" en tanto que sus formas de crecer y acumular se sustenten también en el aprovechamiento de la dinámica del ecosistema. En este senlido, los incremenlos de productividad en alimentos basados en abonos orgánicos, uso de la biomasa, biolecnologia, elc., aparecen como "más seguros" que los conseguidos a base de abonos químicos, mecanización, elc.

\section{Cualidad/consumo apropiado}

Este aspecto cualitativo de la seguridad alimentaria tiene que ver con 
el consumo apropiado de los alimentos. Al menos tres faclores pueden ser considerados en dicho tipo de consumo: la calidad de los productos alimenticios (valor nutrilivo, grados de contaminación, nivel de adecuación al organismo humano, composición tóxica, elc.), la calidad de la dieta (nivel de diversidad, complementariedad o compatibilidad entre los alimentos que la conforman), y finalmenle lo adecuado de los patrones de consumo al medio nacional ("grados de compatibilidad" o de sintonía con la cultura e historia local, con el medio geográfico y climatológico, con el tipo de estruclura económica, con la conformación pedológica, etc.). En tal sentido, podemos formular que a mayor consumo apropiado mayor seguridad alimentaria. Si el consumo es inapropiado (si buena parte de los alimenlos se encuentran contaminados, si la diversidad de la diela es muy pobre, si se demandan consumos que atentan contra la esiructura productiva o que tienden a delormarla), entonces nos alejamos de la seguridad alimenlaria. Podrá suceder, por tanto, que tengamos sulicientes alimentos, accesibles a la población y en un conlexlo de eslabilidad, y, sin embargo, nos vayamos aproximando a la inseguridad alimentaria.

Una vez definidos los componentes que conlormarán nuestra concepción de SA, se hacen necesarias cuatro advertencias:

- Como se habrá notado, los tres primeros parámetros ( $y$ en buena parte el cuarto) son más tácilmente cuantificables, y por ende verilicables. Sobre los dos últimos parámetros (suslentabilidad y consumo apropiado), de carácler más cualilalivo, se encuentra poca información disponible. Ello es normal dada la supremacia de entoques cuantilativos y/o de concepciones que limitan la SA a la cantidad de alimentos, a sus niveles de precio $y$ al poder de compra de los consumidores. En consecuencia, el análisis o evaluación de la SA bajo los dos últimos parámetros se realizará en buena medida a través de planleamientos hipotéticos.

- Dado el carácler limitado y preliminar de este estudio, no se hará un análisis de todos los parámelros de la SA. Nos restringiremos, dentro de los que podríamos considerar como "parámetros más cuantitativos", a la problemática de la suliciencia/disponibilidad alimentaria. Ampliaremos y complementaremos el estudio con el análisis de los parámetros considerados de carácler más cualilativo, los cuales se refieren a la suslentabilidad/viabilidad ecológica y a la calidad/consumo apropiado de alimentos.

- Cuando analizamos el impaclo que la política económica ha tenido en la determinación de los rasgos de la SA en nuesiro pais, no eslablecemos relaciones simples y unilaterales. Aunque aparece como algo 
evidente, es necesario sef́alar que los contenidos que posee la SA en EI Salvador no son producto exclusivo de la política económica. Esta es una dentro de otras mucha causales.

- Dentro de los bienes alimenticios, los esludios y las fuenles estadisticas consultadas destacan los granos básicos. A tal punto que en muchos de ellos la SA aparece casi como un análisis de la' seguridad alimentaria en granos básicos. A pesar de que la imporlancia de estos en la diela $o$ en el consumo de los salvadorefios pueda hacer comprensible tal restricción, creemos que la problemálica alimentaria va mucho más allá de la problemática de los granos básicos. Si nosotros caemos también en el error o deficiencia de centrar nuestros análisis en estos úllimos produclos, no es por falta de conciencia de la limitación, sino de carencias informativas sobre otros productos y abundancia/accesibilidad informativa sobre los granos básicos.

- Sefialada la importancia del tema que nos ocupa y el objelivo general de este estudio, delimilada nuestra noción de seguridad alimentaria y anotadas algunas advertencias al trabajo, podemos pasar a detallar dicho objetivo y a exponer la eslruclura dada al presente documento.

En términos más específicos, el objeto de esta investigación es conocer los efeclos que han tenido determinadas políticas económicas en la contormación de algunos de los rasgos que asume la SA en EI Salvador. Asi, se pretende analizar las incidencias que han tenido las políticas económicas más representativas de los programas de estabilización y ajuste estructural (cambiaria, comercial, fiscal, financiera, de precios e institucional), sobre la suficiencia/disponibilidad alimentaria, por un lado, y por olro, sobre los parámetros de carácter más cualitativos de la SA (sustentabilidad y calidad/consumo apropiado de alimentos).

Acorde a esos objelivos, el trabajo se ha esiruclurado de la siguiente manera. En la primera parte se estudian las caracteristicas y problemáticas concernientes a las disponibilidades alimentarias en El Salvador, y posteriormenle el rol que han jugado en su conliguración las políticas económicas antes mencionadas. En la segunda parte se realiza el análisis, por un lado, de la problemálica que presenla en nuestro país la sustenlabilidad en la dinámica alimentaria, y, por olro, la problemática que se plantea con la calidad y consumo de los alimentos: luego pasamos a definir el papel que han tenido aquellas políticas económicas en la gestación y desarrollo de lales problemáticas.

Para terminar valga anadir que la investigación del resto de parámetros y/o la superación del rango hipotélico de los "análisis cualitativos", está dentro del panorama de nuestros fuluros esfuerzos invesligativos. 


\section{Suficiencia/disponlbilidad allmentaria y las politicas económicas predominantes en El Salvador}

1. La problemática de la disponibilidad allmentarla en EI Salvador: una producción precaria y una olerta Insuliciente de alimentos

\subsection{La precariedad de la producción alimentarla}

La suficiencia/disponibilidad alimentaria tiene que ver (adicionalmente a los aspectos de demanda) con las condiciones en que se produce la oferla alimentaria y con el volumen mismo de la olerta.

En cuanto a las condiciones de producción de alimenlos en El Salvador, podemos afirmar que ésta se ha caraclerizado por su precariedad. Este rasgo podemos analizarlo, enlre olros, a partir de variables como los niveles de precio, el comporlamiento de los coslos de producción, la dinámica de los rendimienlos, los niveles de rentabilidad de los cultivos alimenticios y la disponibilidad de lierras aptas. Nos limitaremos al análisis de las condiciones de producción de los alimentos más importantes para el consumo de los salvadoreños, es decir, los granos básicos.

\subsubsection{Bajo nivel de preclo de los productos}

Durante la década de los ochenla, e incluso para el periodo de liberalización de precios 1989-1991, los precios de los granos básicos se han mantenido relativamente bajos, siendo un desestímulo al incremento de la producción de alimentos y/o a la mejora de la capacidad producliva de los mismos.

Para los producios alimenlicios básicos el cuadro 1 y el grático 1 muesıran la evolución negaliva que han tenido los precios reales al produclor de 1983/84 a 1990/91 (menos para el caso del frijol y más evidente para el caso del producto más importanle de ellos, es decir el maiz; 'hay que destacar, sin embargo, el signilicativo incremento de precios reales del Irijol y arroz de la cosecha $89 / 90$ a la de 90/91). Otras fuentes proporcionan datos que muestran un mayor delerioro en los precios reales pagados al productor de granos básicos, incluso en los produclos que se supone luvieron mejores precios. Asi, CEPAL-CADESCA (1991, p. 10) sefala que si a principios de la década los precios del arroz oro poseian índice de precios cercano a 100, para lines de la década el índice de precios reales redondeaba apenas los 50 . El índice de precios reales en frijol luvo una evolución semejante. 


\section{Cuadro 1}

Indice de preclos de los granos básicos a nivel de produclor $e$ indlces de precios de los insumos utilizados en su producción $(1976 / 1977=100)$

\begin{tabular}{|c|cc|rc|cc|rr|}
\hline \multirow{2}{*}{ AÑO } & \multicolumn{2}{|c|}{ MAIZ } & \multicolumn{2}{c|}{ FRLOL } & \multicolumn{2}{c|}{ ARROZ } & \multicolumn{2}{c|}{ SORGO } \\
\cline { 2 - 9 } & IPP & IPIU & IPP & IPIU & IPP & IPIU & IPP & IPIU \\
\hline $1976 / 1977$ & 100.0 & 100.0 & 100.0 & 100.0 & 100.0 & 100.0 & 100.0 & 100.0 \\
$1977 / 1978$ & 119.4 & 146.7 & 102.0 & 106.4 & 115.0 & 93.9 & 116.7 & 94.3 \\
$1978 / 1979$ & 112.5 & 169.3 & 92.5 & 113.9 & 109.6 & 84.0 & 107.9 & 84.5 \\
$1979 / 1980$ & 104.0 & 207.9 & 104.2 & 169.0 & 104.5 & 119.7 & 114.7 & 128.6 \\
$1980 / 1981$ & 114.8 & 297.8 & 134.2 & 280.5 & 108.4 & 170.4 & 129.1 & 156.7 \\
$1981 / 1982$ & 127.4 & 353.5 & 146.4 & 337.8 & 113.4 & 198.7 & 135.2 & 267.0 \\
$1982 / 1983$ & 152.4 & 343.3 & 11.2 & 337.8 & 125.0 & 189.0 & 145.0 & 259.5 \\
$1983 / 1984$ & 164.0 & 278.4 & 102.6 & 330.3 & 125.0 & 148.4 & 143.2 & 258.7 \\
$1984 / 1985$ & 154.9 & 353.6 & 117.3 & 203.5 & 116.6 & 138.2 & 137.5 & 228.8 \\
$1985 / 1986$ & 191.6 & 313.7 & 147.3 & 220.0 & 128.9 & 153.9 & 148.8 & 260.7 \\
$1986 / 1987$ & 231.6 & 535.0 & 163.3 & 343.0 & 147.3 & 318.5 & 201.6 & 443.8 \\
$1987 / 1988$ & 236.6 & 502.0 & 219.6 & 300.5 & 178.4 & 266.9 & 232.6 & N.D. \\
$1988 / 1989$ & 281.5 & 395.5 & 316.7 & 469.8 & 206.5 & 284.4 & 267.0 & 291.7 \\
$1989 / 1990$ & 308.1 & 434.7 & 297.9 & 407.4 & 162.3 & 334.5 & 275.0 & 298.3 \\
$1990 / 1991$ & 351.3 & 541.0 & 387.3 & 530.9 & 254.2 & 396.4 & 330.8 & 384.0 \\
\hline
\end{tabular}

IPP = Indices de precios del producto

IPIU = Indice de precios de los insumos ulilizados

Fuente: Tomado de Pleilez, W. (1991, Cuadro 52, p. 97).

En el cuadro 2 puede apreciarse en forma global el deterioro de los precios de los granos básicos respecto al comportamiento de los precios de los bienes en general (precios al consumidor).

\subsubsection{Altos costos de producción}

La siluación de los cosios de producción tampoco ha sido favorable a los productos agrícolas básicos. Si los precios se han caracterizado por su nivel relativamente bajo, los costos de producción lo han hecho por su nivel relativamenle allo. Eslo puede percibirse en los anexos 1, 2, 3 y 4, donde los coslos de producción de los granos básicos han sido normalmente mayores que los ingresos tolales. Es más, en determinados, y a veces prolongados periodos, los coslos de producción fueron siendo cada vez mayores que los ingresos (de 1983 a 1987 en maiz, sorgo y arroz). 


\section{Cuadro 2}

El Salvador: Indice de reclos de los granos báslcos entre indices de preclos al sonsumldor (en porcentajes) 1976/1977 = 100

\begin{tabular}{|c|r|r|r|r|r|}
\hline AÑO & IPC & IPM/IPC & IPF/IPC & IPAIPC & IPS/IPC \\
\hline $1976 / 1977$ & 100.0 & 100.0 & 100.0 & 100.0 & 100.0 \\
$1977 / 1978$ & 111.8 & 106.8 & 91.2 & 102.9 & 104.4 \\
$1978 / 1979$ & 126.7 & 88.8 & 73.0 & 86.5 & 85.2 \\
$1979 / 1980$ & 146.8 & 70.8 & 71.0 & 71.2 & 78.1 \\
$1980 / 1981$ & 172.2 & 66.7 & 77.9 & 63.0 & 75.0 \\
$1981 / 1982$ & 197.7 & 64.4 & 74.1 & 57.4 & 68.4 \\
$1982 / 1983$ & 220.9 & 69.0 & 50.3 & 56.6 & 65.6 \\
$1983 / 1984$ & 249.9 & 65.6 & 41.1 & 50.0 & 57.3 \\
$1984 / 1985$ & 279.1 & 55.5 & 42.0 & 41.8 & 49.3 \\
$1985 / 1986$ & 341.4 & 56.1 & 43.1 & 37.8 & 43.6 \\
$1986 / 1987$ & 450.5 & 51.4 & 36.2 & 32.7 & 44.8 \\
$1987 / 1988$ & 616.1 & 38.4 & 35.6 & 29.0 & 37.8 \\
$1988 / 1989$ & 723.9 & 38.9 & 43.7 & 28.5 & 36.9 \\
\hline
\end{tabular}

Fuente: Tomado de Pleitez, W. (1991, cuadro 53, p. 99).

\subsubsection{Carencla de rentabilldad}

Como lo podemos apreciar en el cuadro 3, el resultado del comportamiento de precios y costos recién analizado ha sido la falta de rentabilidad que posee la producción de los alimentos básicos. La rentabilidad del maiz y maicillo desde $1976 / 77$ al presente ha sido siempre muy negativa ( $y$ en general podemos hablar de una rentabilidad con lendencia decreciente). Sólo podemos hablar de cierta recuperación punlual de la rentabilidad, para las cosechas $88 / 89$ y $90 / 91$ en los casos del frijol y el arroz.

\subsubsection{Deterioro de los rendimlentos}

Como causa y efecto de la falta de rentabilidad de los granos básicos encontramos un deterioro en los rendimientos de los mismos. Así, si en la década de los 70 , los rendimientos promedio en granos básicos crecieron en $45.2 \%$ para el cultivo del maiz, $25.8 \%$ para el caso del Irijol y $31.1 \%$ en el arroz; en la década de los ochenta bajaron al $12.9 \%$ en el maíz, al $2.1 \%$ en el trijol y $11.1 \%$ en arroz, respectivamente (Pleitez, et. al., p. 26). El gráfico 2 muestra con claridad el práclico eslancamienlo de 


\section{Cuadro 3}

El Salvador: Beneflclos netos obtenidos por el cultivo de una manzana de granos básicos (periodo 1976/1977 - 1990/1991).

\begin{tabular}{|c|r|r|r|r|}
\hline $\begin{array}{c}\text { AÑO } \\
\text { Cosecha }\end{array}$ & $\begin{array}{r}\text { MAIZ } \\
(\notin / \mathrm{MZ})\end{array}$ & $\begin{array}{r}\text { FRIJOL } \\
(\varnothing / \mathrm{MZ})\end{array}$ & $\begin{array}{r}\text { ARROZ } \\
(\notin / \mathrm{MZ}\end{array}$ & $\begin{array}{r}\text { SORGO } \\
(\notin / \mathrm{MZ})\end{array}$ \\
\hline $1976 / 1977$ & -358.29 & 114.19 & -86.29 & -378.51 \\
$1977 / 1978$ & -263.31 & 223.25 & 256.92 & -242.82 \\
$1978 / 1979$ & -303.15 & 72.51 & 558.77 & -266.63 \\
$1979 / 1980$ & -449.17 & -16.60 & -425.92 & -354.11 \\
$1980 / 1981$ & -682.25 & -292.12 & -6.13 & -330.63 \\
$1981 / 1982$ & -702.40 & 294.55 & -2.32 & -735.62 \\
$1982 / 1983$ & -614.12 & -624.54 & -39.60 & -759.41 \\
$1983 / 1984$ & -466.56 & -610.73 & 219.63 & -816.09 \\
$1984 / 1985$ & -632.95 & -94.72 & 503.27 & -831.42 \\
$1985 / 1986$ & -459.41 & -229.89 & 542.41 & -840.61 \\
$1986 / 1987$ & -963.99 & -201.08 & -354.30 & $-1,143.14$ \\
$1987 / 1988$ & -731.12 & -539.32 & 154.15 & $N . D$. \\
$1988 / 1989$ & -44.10 & 567.42 & 639.55 & -386.21 \\
$1989 / 1990$ & -165.77 & 181.65 & -619.74 & -394.50 \\
$1990 / 1991$ & -381.32 & 833.04 & 735.51 & -445.52 \\
\hline
\end{tabular}

Fuente: Pleitez, W. (1991, cuadro 50, p. 91).

los rendimientos del cultivo de granos báscos durante la década de los ochenta y principios de los noventa.

\subsubsection{Poca y mala tlerra}

Otro problema relacionado con la precariedad en la que se desarrolla la producción de alimentos básicos es la poca o inadecuada disponibilidad de tierras. En primer lugar, llama la atención el estancamienlo regisIrado también en el incremento de la superficie destinada a dichos cultivos: desde $1978 / 80$ a 1990/91 la superticie lolal de granos básicos apenas aumentó en 18 mil manzanas aproximadamente (Cruz Letona, 1991, p. 10). El Grálico 3 revela con contundencia el estancamiento en los incremenlos de superticie destinada a granos básicos desde 1976/77. Además, irente a los ligeros o insignificantes incrementos de superticie ocurridos, hay que tomar en cuenta que "la superticie cosechada de granos básicos aumentó, pero eslos disminuyeron su capacidad produc- 
tiva -a excepción del arroz-, puesto que utilizaban una mayor proporción de lierras marginales" (Arroyo y Arias, 1987).

En segundo lugar, la inadecuada disponibilidad de tierras dedicadas al cultivo de granos tiene que ver con el tamaño y calidad de las mismas. En este sentido, la producción de los bienes agricolas básicos se carácteriza por desarrollarse en tierras de poco lamafio y mala calidad. Concerniente al primer aspecto, traigamos a cuenta el hecho que casi un $55 \%$ de la superficie cultivada con tales granos se hace en explotaciones menores de 5 hectáreas (Pleitez, W., p. 51). En cuando al segundo aspeclo, indiquemos que "el cultivo de granos básicos en El Salvador no solamente eslá concentrado en las explotaciones más pequefias, sino lambién en los suelos de menor calidad. Prueba de ello es que del área lotal cultivada con granos básicos durante el año agrícola 1983/84 único para el cual fue posible obtener información-, sólo un $20.2 \%$ perlenecía a las tierras clases I, II y II, que son aquellas de mayor calidad y aptas para una agricultura intensiva y mecanizada, con pocos riesgos de erosión. La mayor parte $(50.4 \%)$ eran tierras clases VI y VII, las cuales se caracterizan por ser suelos de alta pendiente y poca vocación agrícola" (Pleitez, W., p. 72).

En conclusión, bajos precios, allos costos, poca/nula o negativa rentabilidad, deterioro o estancamiento de los rendimientos, disponibilidad baja o inadecuada de tierras, son todos ellos faclores que contribuyen a conformar unas condiciones de producción precarias para importantes bienes alimenticios.

\subsection{Una oferta alimentaria insuficiente}

La insuficiencia alimentaria podemos valorarla tanto en términos de producción nacional como en lérminos generales.

\subsubsection{La insuficlencia de la producción nacional}

A nivel de la producción nacional, la oferta alimentaria se vuelve insuficiente en la medida que contribuye cada vez menos a la salisfacción de las necesidades alimenticias de la población. Es decir, la producción nacional de alimentos será insuliciente en la medida que el consumo nacional de alimentos se satisfaga con mayores importaciones. Al respecio, todos los dalos recabados apuntan a un allo y creciente nivel de importación de alimenlos desde principios de los años 80 (las iniormaciones aparecen más adelante, en la sección 3 de esta parte). Por ejemplo, es noloria la pérdida reciente de suliciencia en la producción nacional de aceites comeslibles, que ha llevado a El Salvador a convertirse en el principal importador de aceiles dentro de la región centroamericana. 
La insuliciencia alimentaria se acrecienta cuando consideramos los requerimienlos fuluros de alimentos. Tomando en cuenta los requerimientos nacionales para el ano 2000 , tenemos que para cubrir la demanda polencial de granos básicos en este año, la superticie existenle de los mismos en 1985 deberia haberse casi duplicado (de 465 mil hectáreas se debería haber pasado a las 845 mil hectáreas; esı bajo el supueslo de una lasa de crecimiento constante en los rendimientos); lo que implicaría una lasa de crecimiento del área cullivada del $3.8 \%$ anual, mienlras que dicha tasa el periodo 1950-1984 apenas lue del 1.3\% anual. Asimismo, las tasas medias de crecimiento de los rendimientos en la producción de maiz, frijol y maicillo, que en el periodo 1950-84 fueron de apenas $1.5 \%, 0.2 \%$ y $0.4 \%$ anual, respectivamente, para satisfacer dicha demanda potencial demanda potencial deberán alcanzar las tasas medias del $4.2 \%, 4.2 \%$ y $5.4 \%$ durante el periodo $1985-2000$ (AID, et al pp. 460-61).

Creemos necesario advertir que una producción nacional suliciente puede ser compatible con una siluación alimentaria precaria. En efeclo, podemos encaminarnos hacia un producción alimenticia suticiente (por medio de los aumentos de producción de alimentos) y, sin embargo, podriamos dirigirnos hacia un deterioro en las condiciones de producción y en el consumo de alimentos. ¿Cómo es eslo posible?

De hecho, la producción de alimentos de un pais puede experimentar sensibles incremenlos $y$, sin embargo, no afiadir ningún bienestar a la población. Más aún, como lo constatarian Collins y Lappe (1986), en varios paises (Filipinas, Bangladesh, Malasia Occidental y Sri Lanka) el incremento de la pobreza es compalible con los aumenlos percápila de los granos básicos: "un mayor volumen de alimentos puede servir para alimentar menos gente... el aumento de la pobreza no se asocia con una caída, sino con un aumento en la producción de granos percápita, componente principal de la dieta de los pobres" (Lappe y Collins, 1986, pp. 135 y 152).

Lo anterior puede ser más comprensible cuando consideramos que puede haber crecimiento de la producción alimentaria, pero una parte puede deslinarse a la exportación, o puede suceder que "una parte se destina a los grupos urbanos de ingresos altos y medios.... Una parte se convierte en produclos de lujo que los pobres no pueden adquirir (como la conversión de maiz en Corn Flakes. $\mathbf{N}$. del A.).... una parle se destina a la engorda del ganado para producir carne que la mayoría de la población local no puede adquirir". (Collins y Lappe, pp. 152-3). He aqui por qué una evaluación de la siluación alimentaria debe preguntarse adicionalmente por la accesibilidad o demanda de los bienes alimenli- 
cios, por su deslino económico o social, por el tipo de transformación industrial a que se someten.

Por otro lado, los mismos incrementos de la producción de granos pueden ir en contra de los mismos campesinos que los producen. Un incremento de la oferta puede significar una disminución de los precios de los bienes alimenticios y aleclar negativamenle a los campesinos pobres, sobre todo cuando tal incremento es llevado a cabo por los campesinos medios, como es normalmente el caso (que se verifica en la siluación salvadorefia). Asimismo, mayores aumenlos de producción de granos no signilican mayores ganancias para sus productores campesinos; éslo en razón de que, como es también el caso normal y puede ilusirar el caso salvadoreño, el sislema de comercialización que controlan los mismos comerciantes o usureros se apropia de una parte significativa del excedente, que podría haber quedado en manos de los mismos productores.

Finalmente, como se sef́ala en Collins y Lappe (1986, p. 135), en sistemas productivos "en que se permile que los recursos agricolas sean luente de riqueza privada, el mecanismo para aumentar la producción alimentaria ha empeorado las condiciones de vida de la mayoría pobre, a pesar de los incrementos en el produclo percápita". Los autores sefialan algunas consecuencias que se derivan de las formas de llevar a cabo el crecimiento en la producción de alimentos: elevación del precio de la tierra y expulsión de los arrendalarios y pequefíos productores, incremento de la renta de la tierra, mayor conlrol de la tierra en pocas manos, mayor presencia de la inversión extranjera en la eslera de la producción, empeoramiento de la situación de las mujeres, aumento del endeudamiento campesino, "la cantidad y el valor mercantil, no el valor nutritivo, se convierten en el objetivo final de la planificación agricola" (p. 135).

\subsubsection{Disponlbilidades alimentarlas Insuficlentes}

A nivel general, la suliciencia alimentaria se valora en términos de disponibilidades. Las disponibilidades comparan la oferla global de alimentos disponibles para consumo humano (producción más importaciones) con la demanda polencial de alimentos de la población.

Antes de conocer la situación de las disponibilidades alimenlarias en El Salvador, es menesler hacer algunas observaciones sobre su cálculo.

En primer lugar, del lado de la oferla:

- Hay fuentes inlormalivas que calculan las disponibilidades en base a la oferta lolal de alimenlos (inventarios + producción nacional + importaciones). Esto sobreestima las disponibilidades, ya que debe conside- 
rarse sólo la olerla disponible al consumo humano nacional. Es decir, a la oferta total habria que deducirle las exporlaciones, las pérdidas postcosecha, los alimentos usados como semillas o materias primas para procesos industriales, los deslinados al consumo animal.

- Por otra parte, la oferta total puede ser subestimada. Esto puede suceder cuando en el cálculo de los balances que hacen las instituciones públicas - como es el caso de la Dirección General de Economía Agropecuaria (DGEA) - sólo se conlabilizan las existencias públicas de granos y se omiten las que se encuentran en manos privadas. Además, la subestimación de la oferla se da por la lalta de registro que acompana el contrabando.

Por el lado de la demanda, encontramos las siguientes limitaciones:

- Muchas disponibilidades alimentarias se establecen sobre la base de la demanda efectiva y no de la demanda polencial. Esto es equivocado, ya que se trala de conocer los alimentos que se encuentran disponibles para loda la población y no sólo para aquellos que poseen poder de compra.

-Otra deficiencia estriba en que se suelen calcular las demandas inlernas a partir de diferencias (lo producido menos lo invertido). Expuestas algunas carencias que normalmenle acompanan las informaciones sobre disponibilidad alimentaria, pasemos a dar una visión global de la siluación de ésta en nuestro pais.

Según Pleitez et. al. (1991, p. 119), quien estima las disponibilidades en granos básicos de la oferla total (sin introducir las observaciones anleriores), en la década de los ochenta las disponibilidades de maiz en El Salvador se consideraban suficientemente para cubrir la demanda potencial. No era así el caso del frijol y arroz. La oferla lotal de esios productos apenas cubrian el $38.5 \%$ y $47.6 \%$ de la demanda potencial (como promedio de la década).

Pero la situación de la disponibilidad alimentaria (de granos básicos) se empeora cuando lomamos en cuenta algunas de las observaciones hechas. Asi, si sólo consideramos la olerta disponible para consumo humano, la siluación de supuesta suliciencia desaparece. Según datos oficiales (FUSADES y DGEA) usados por Cruz Lelona (1991, pp. 23 y 68), para estimar los requerimientos de consumo de granos en 1990, la demanda de maíz era aproximadamente de 11.1 millones de quintales; mientras que por el lado de la olerta para consumo humano, durante los afios $89 / 90$ y $90 / 91$ (de agosto a julio), la oferta de maiz se mantenía en 10.3 millones de quintales (sólo un 93\% de lo requerido, es decir, "una insuficiencia" del 7\%). 
Ahora bien, las insuliciencias en las disponibilidades no se presentan sólo a nivel de los granos básicos, sino también a nivel de los produclos alimenticios en general. Esto podrá ser coslatado a través de los llamados Balances de Disponibilidades Alimentarias. Desgraciadamente, solamente existen dos Balances, uno para 1965 y otro más completo para 1975-1982 (véase Pleitez, W., pp. 8-12). En el balance de 1965 el déficil en disponibilidades alimenlarias es claro: siendo los requerimienlos recomendados por INCAP de 2048 calorias y 57 gramos de Proleina, los alimentos percápita disponibles en ese ańo sólo proporcionaban 1928 calorias y 47.6 gramos de proteína. Para 1975, las disponibilidades alimenlicias habia mejorado, ya que eslas contenian 57.4 gramos de proteína y 2688 calorias percápila, es decir, valores un poco superiores a los recomenddaos a la época. Llama la atención que a partir de 1979 las disponibilidades alimenlarias comienzan a sutrir un franco deterioro, llegando en 1982 a proporcionar apenas 43.4 gramos de proteina (menos que en 1965) y 2057 calorías percápila.

Es de suponer que con la profundización de la crisis socio-económica y militar que siguió a 1982 las disponibilidades alimenticias hayan seguido disminuyendo, a tal punto que para tines de la década de los ochenla quizá podemos hablar de graves insuliciencias alimentarias. "Lamentablemente, después de 1982 se dejaron de construir las hojas de balace. pero no cabe duda que, tal como lo confirman otros indicadores, la situación alimentario-nutricional se haya continuado agravando lodavía más como consecuencia de la profundización de la crisis socieconómica" (Pleilez, W., p. 12).

En resumen, he aqui una caracterización de uno de los parámetros que miden la seguridad alimentaria de nuestro pais: condiciones precarias en la producción alimenticia (bajo precios, allos cosios, poca/nula o negativa rentabilidad, deterioro o freno de los rendimientos, disponibilidad baja o inadecuada de tierras), una producción nacional de alimentos cada vez más insuficientes, fuertes délicils en la disponibilidad de alimentos, e incluso cierta falla de disponibilidad en el produclo alimenticio más signilicativo e importante para el consumo de los salvadorefios, es decir, el maiz. ¿Cuál es el rol que la política económica dominante ha jugado en todo ello?.

\section{Incidenclas de la politica económica en la disponlbllidad alimentarla de El Salvador}

\subsection{Varlables determinantes de la disponlbilldad allmentarla}

Para ver de que manera las políticas económicas predominantes han 
incidido en los problemas de disponibilidad antes descritos, es necesario conocer previamente las variables de las que ésla depende.

Con el objelo de tener un marco de referencia sobre las variables que determinan la disponibilidad alimentaria en nuestro pais, hemos elaborado la siguiente ecuación: ${ }^{2}$

$$
D A=1(P A+P F+D R+1+C R+R A+M-D P) .
$$

donde:

DA = Disponibilidad alimentaria, la cual compara la oferta total de alimenlos, producida e imporlada, con la demanda polencial de los mismos.

$\mathrm{PA}=$ Precios de los alimentos

$\mathrm{PF}=$ Precio de lo factores de producción. Tomamos como 'Hactores de producción" el trabajo, el capilal (financiero y productivo) y la tierra. Por lanto, los precios de dichos factores se refieren a los salarios, la lasa de interés, la tasa de ganancias o rentabilidad, la renta de la tierra.

DR = Disponibilidad de recursos físicos, es decir, las cantidades o volúmenes de fuerza de trabajo, tierra/agua, insumos, maquinaria y equipo, instalaciones, crédito.

I = Disponibildad de intraeslruclura de circulación. Comprende la disponibilidad en inlraestruclura de almacenamiento, conservación, Iransporte y comercialización-distribución de alimentos.

$\mathrm{CR}=$ Estado o calidad de los recursos e inlraestruclura fisica. Acá nos relerimos básicamente a la productividad de la fuerza de trabajo (la cual a su vez liene que ver con elemenlos como el eslado de salud y educación de los (rabajadores), la calidad del ecosistema o estado del capital natural (grado de delerioro ecológico, por ejemplo), el tipo de insumos, máquinas y equipos ulilizados (equipos de buena calidad 0 apropiados, fertilizantes químicos u orgánicos, elc.), caracterislicas que posee el crédito, calidad y lo apropiado de la iniraestructura básica relacionada con el sistema alimentario (estado de carreteras, silos, obras de

2. Como la relación de las variables mediante ecuaciones no tiene como fin la medición, sino el de la ilustración de las relaciones que ahi se establecen, es pertinente aclarar que no se prelende agolar las variables independienles, sino sólo colocar las que consideramos más importanles. 
irrigación, mejoras en tinca, distribución espacial de centros de acopio, distribución o compra-venta de alimentos, etc.).

RA = Disponibilidad y calidad de los recursos de apoyo básico . Como "recursos de apoyo básico", podemos mencionar la asislencia técnica, la capacitación y la investigación.

$M=$ Importaciones de alimenlos. Donde se tomará en cuenta no sólo los volúmenes importados legalmente, sino lambién las posibles eslimaciones que pudieran hacerse de los bienes alimenlicios que entran vía contrabando.

DP = Demanda potencial de alimentos. Esta demanda depende a su vez de variables como la cantidad de población, la lasa de crecimiento y composición de la misma, tipos y estilos de Irabajo, lipos y estilos de vida y de consumo.

De las primeras seis variables depende la capacidad nacional de producción de alimentos. Así, este conjunlo de variables más la variable $M$ conforman la oferta total de alimentos. Si a esta oferta le restamos la demanda potencial de alimentos o DP, entonces tendremos determinada la disponibilidad alimentaria, DA.

\subsection{El rol de las politicas económicas predominantes en la conflgu- raclón de los problemas de dlsponibilldad alimentarla en EI Sal- vador}

Como se habrá notado, pretendemos conocer las incidencias de la política económica sobre la problemática de las disponibilidades alimentarias, a Iravés del impacto que dichas polílicas han tenido sobre las variables que determinan la disponibilidad alimenlaria. Evidentemente, en un trabajo de objetivos limitados como el nuestro, no podemos analizar las incidencias de las políticas económicas sobre todas las variables recién expuestas. Hay que hacer una selección de éstas. En este sentido, concentramos el análisis en aquellas variables en las que hemos obtenido más información. Asimismo, no estudiamos el impaclo de la política económica sobre algunas de las variables que, como la calidad del ecosistema, son abordadas más adelante, dentro de los otros parámetros de la seguridad alimentaria (la sustentabilidad, para el caso).

\subsubsection{Efectos de la polítlca camblarla}

Lo que ha caracterizado a la polílica cambiaria, tanlo antes como después de 1989, ha sido la tendencia hacia la sobrevaluación de la moneda nacional. A grosso modo, la principal incidencia de ello es el abaratamiento de las importaciones, de granos para el caso, y en conse- 
cuencia la presión a la baja en los precios de los granos básicos en el mercado nacional. Para tener una idea global del impacto de la sobrevaluación sobre los precios reales de los granos básicos, Iraigamos a cuenta una cita que revela ese impacto a nivel de los bienes agropecuarios en general: "un esludio reciente estimó que... en la caída de los precios reales agropecuarios, el $53 \%$ de ello fue determinado por la sobrevaluación cambiaria" (Cruz Lelona, 1991, p. 24) Como veremos más adelante, el abaralamiento e incremento de las importaciones de granos contribuyó lambién la política comercialarancelaria.

Es pertinente destacar que la sobrevaluación cambiaria que se experimentó en El Salvador, en especial antes de 1989, fue de las más allas a nivel centroamericano (como lo lue lambién la desprolección arancelaria). Por ello no lue casual que una investigación de CADESCA sefialara que "El Salvador tue el país del Isımo que imponó más granos -entre 200,000 y 250,000 toneladas en 1983 y 1984 , que coincidió con el mayor grado de sobrevaluación" (CEPAL-CADESCA, p. 43).

Curiosamente, la menor sobrevaluación cambiaria que se da a partir de la puesta en marcha de los Programas de Eslabilización Económica y Ajuste Estruclural (PEE/PAE) en 1989, no parece haber contribuido al ireno y/o abaralamiento de las importaciones de granos. Asi, sólo las imporlaciones de cereales y frijol ocurridas en 1989/90 y $90 / 91$ fueron superiores a todas las importaciones habidas desde 1979/80 a 1985/86 (Pleitez, W., 1991, cuadros anexos 27 y 20). Eslo podia ser explicado en parte por la política de comercialización y/o arancelaria aperturisla, que luego del 1989 impulsan los PEE/PAE en nuestro pais.

Ahora bien, según lo dicho arriba podría argumentarse que la sobrevaluación cambiaria puede afectar la producción local vía precios, pero no afecta la disponibilidad. En efeclo, aunque la producción nacional baje la oferta global podrá manlenerse o incrementarse vía imporlaciones; éstas ahora más baralas. Estos mismos argumentos sirven a muchos defensores de los PEE/PAE para sostener la conveniencia de ir sustituyendo laproducción nacional de granos básicos (ineliciente, con allos costos y precios) por imporlaciones (más baralas). Con ello el consumidor en general se benelicia. A su vez, se sigue argumentando, por medio de una política de "reconversión agrícola" hacia la exportación de miles de pequefios y medianos productores, se podrá enirenlar adecuadamente las necesidades de divisas que las crecientes importaciones de granos irán demandando.

Los argumentos anleriores nos parecen inadecuados por lo siguienle:

-Es cierto que las disponibilidades alimentarias no son necesariamenle afectadas por la sobrevaluación cambiaria, pero si es claramenle 
afectado en forma negativa el nivel de lo que definiamos como "suficiencia de la producción nacional". Esta, aunque no define por si los problemas de disponibilidad alimentaria, es un componente importante a tener en cuenta en la misma. Además, la suticiencia de la producción nacional es un factor determinante en otro de los parámetros que miden la SA, como es la autonomia/vulnerabilidad.

-Más allá de los criterios de afectación de la SA, una política de sustitución de producción nacional de granos por importaciones no es tan adecuada económicamenle hablando. Primero, porque significa "sustituir" a miles de pequeños y medianos produclores (los que junto con sus familiares hacen aproximadamente más de 1.2 millones de personas). Evidentemente, "reconvertir" el complejo mundo de más de un millón de campesinos no será cosa lácil, e implica una política de alto riesgo económico, social y político. En segundo lugar, porque la SA se deja mucho en manos de las divisas de posibles exportaciones. Esto no deja de ser arriesgado frente a un mercado mundial de alimentos controlado por pocas compañias transnacionales y con tendencias actuales depresivas, frente a los crecientes riesgos de mantener "venlajas comparativas esláticas" (sobre todo en bienes agropecuarios), y de cara a las inestabilidades que se dan en el comercio exterior. Respeclo a este último punto, "cabe agregar una consideración sobre los lérminos de Intercambio entre los cullivos tradicionales de exportación y los precios CIF de los granos básicos. En general, su margen es pequeño, pero acusa sobre todo variaciones significativas de un año a otro, lo que resta seguridad alimenlaria por la via de las importaciones" (CEPAL-CADESCA, 1991, p. 45).

Por otra parte, los detensores de los PEE/PAR consideran que la sobrevaluación cambiaria tuvo efectos positivos sobre la disponibilidad alimentaria, vía abaralamiento de los insumos. Otros razones nos llevan a pensar que ello no es tan cierlo. En primer lugar, los cosios de producción, en especial de los insumos, lejos de bajar subieron. E incluso subieron más que los precios de los alimentos/granos básicos. Una prueba de ello es el deterioro en los términos de intercambio de los precios de los granos básicos respecto al precio de los insumos desde 1980 hasta 1991 (Pleitez, W., cuadro 52, p. 97). En segundo lugar, la sobrevaluación cambiaria puede en efecto bajar el precio de los insumos importantes, pero ello no quiere decir que bajen los precios de venla al productor. En efecto, los insumos pueden ser más baratos para el pequeño grupo que controla el mercado de su distribución, pero estos lo pueden seguir vendiendo más caro a los produclores de alimentos.

Finalmente, habría que añadir que en los últimos años (con los PEE/ 
PAE) la política de sobrevaluación cambiaria tendió a anular los posibles electos benélicos de la polílica de precios del aclual gobierno, en lo que en maleria de Banda de Precios concierne. Si la politica de la Banda de Precios "es una de las pocas áreas en las que el actual gobierno ha realizado importantes avances a nivel sectorial, estos avances han resultado insuficientes para la eliminación del sesgo antiagrícola que tradicionalmente se ha mantenido.... aún no se logra el objelivo de llegar a un tipo de cambio realista, que es el prerrequisilo principal para alcanzar el éxito esperado con la banda de precios en términos de competitividad" (Pleitez, R., pp. 205-6).

Como hemos podido conslatar, un balance global de los efectos de la polílica cambiaria sobre este importante parámetro de la SA, arroja un saldo negativo. Fundamentalmente los efectos se han dejado sentir sobre las variables PA y $\mathrm{M}$ de nuestra ecuación. Los posibles beneficios de la sobrevaluación en el abaratamiento de las imporlaciones de bienes alimenticios e insumos, no están adecuadamente sustenlados.

\subsubsection{Efectos de la política comercial-arancelaria}

En términos generales, la política comercial arancelaria ha deseslimulado la producción nacional de alimentos y, en ciertos casos, reducido las disponibilidades de algunos de ellos.

Antes de 1989, por ejemplo, gran parte de las importaciones de granos, aceiles y leche se hacia sin aranceles a través del IRA o del PL480. Con la implementación de los PEE/PAE luego de 1989, las prolecciones arancelarias vigentes disminuyeron dráslicamente, en especial sobre los granos básicos y otros bienes alimenlicios asociados a la industria de alimentos. A tal punlo que El Salvador, a tines de 1990, disponia, después de Gualemala, de la menor coberlura arancelaria en granos básicos a nivel centroamericano (Cruz Letona, 1991, cuadro 11 , p. 19).

Anles de 1989, un indicador del desestímulo a la producción nacional de alimentos fue la politica de importaciones. Rivera Campos (1988) muestra la correlación que existe entre la disminución de la producción de maiz y el incremenlo de sus importaciones y viceversa, llega a señalar que "en un estudio realizado a fines de la década pasada (Siri y Dominguez, 1979), mostraban que enlre 1960 y 1976 el gobierno salvadoreño se apoyó en los suminislros exiranjeros de granos básicos en un monto promedio equivalenle al $22 \%$ de la producción doméstica y observan que el indice de producción tiende a eslar sislemálicamente correlacionado de manera inversa con la tasa de importaciones en el siguiente periodo" (Rivera Campos, 1988, p. 12). Eslos datos permilen al 
aulor afirmar que el sector extemo, o la política agrícola de importaciones, ha eslado sirviendo como estabilizador de la oferla nacional de maíz, y por ende de sus precios.

Luego de 1989, con la liberalización del comercio exterior y reducción de las tarilas arancelarias se marcan los impaclos de la devaluación inicial (mediados de 1989) sobre el déficil de la balanza comercial. Posteriormente, los mayores grados de desgravación arancelaria se suman a la tendencia a la sobrevaluación del tipo de cambio, para dar por resultado una fuerte promoción de las importaciones. Asimismo, se continuó reforzando éslas bajo el PL-480. Sin embargo hay que reconocer que los intentos de canalizar las donaciones bajo los mecanismo de mercado y/o de evitar la competencia desleal pudo haber tenido un menor efeclo depresivo sobre los precios de los bienes alimenticios. ${ }^{3}$

Dentro del acelerado incremento de las importaciones destacan las de alimentos. Valga señalar que al inlerior de éslas las imporlaciones en granos básicos no han sido las de mayor peso: "dentro de la estructura de imporlaciones de alimentos, las de granos básicos han sido mínimas en comparación con los produclos utilizados por la indusiria alimenticia: trigo, leche, aceile vegetal, grasas y harinas protecas. Esios cinco productos constituyen más de la mitad de las importaciones agrícolas, en comparación con un promedio de menos del 10\% de los granos básicos" (Cruz Lelona, 1991, p. 26)

Respecto al incremento de las imporlaciones de ciertos alimentos, indiquemos dos consecuencias. En primer lugar, las enormes imporlaciones de trigo, como bien sustituto del maiz, podrian llegar a afectar las disponibilidades de este último produclo. El mayor consumo de pan y menor consumo de maiz podría reducir lanto la producción nacional de maiz blanco para consumo humano como las importaciones del mismo. Por ende, las disponibilidades percápila de maiz podrían irse reduciendo. En segundo lugar, las también significativas importaciones de leche en polvo daña la producción del halo lechero y de la ganadería mixla (leche

3. Es irónico pensar que la política de donaciones del gobierno habla de evitar la competencia desleal, cuando su política comercial/arancelaria es en sl desleal e injusta. Porque no es justo ni leal abrir aceleradamente el comercio exterior para poner a competir unos "productos desarrollados" (con altos niveles de productividad, apoyo investigativo, financiero, elc., y para colmo subsidios, como suelen ser los bienes agrlcolas producidos en los palses del Norte) con unos "produclos producto del mal-desarrollo" (bajos niveles de productividad, malas tierras, marginalidad ante servicios de la equidad y de la similitud de condiciones, pero no en el marco de la desigualdad y la injusticia). 
y carne). La actual crisis del seclor lo confirma. Tómese en cuenta que la conversión del halo lechero en hato de engorde para carne no es fácil, en parte debido a la estrechez del mercado nacional y las dilicultades para acceder al mercado externo de carne. Por tanlo, al menos en el caso de la ganadería mixla, el desestimulo a la ganadería de engorde. Con ello no sólo se atenta contra la suficiencia de la producción nacional de carne, sino también conlra las disponibilidades de este producto en general (ya que las importaciones de carne son limitadas, en el marco de un mercado local con bajo poder de compra).

En resumen, la política comercial arancelaria, facililando las importaciones de alimento y contribuyendo a los niveles bajos de precios de los mismos (indiciendo sobre PA y M), se convierte en impulsor del carácter insuficiente de la producción de muchos bienes alimenticios. Por otro lado, las mismas ventajas que para las disponibilidades alimentarias representan las facilidades de importación, pueden llegar a convertirse en desventajas para las mismas, al menos para los casos analizados del maiz y la carne.

\subsubsection{La politica de comercializaclón Interna y precios ${ }^{4}$}

En el período anterior a la puesta en marcha de los PEE/PAE, la política de control de la comercialización interna de los alimentos, en especial de granos básicos, se orientaba al mantenimiento de un nivel bajo en los precios de los mismos, tanto a nivel de productor como de consumidor. ${ }^{5}$ Con la llegada del gobierno de ARENA, el paso a la liberalización del comercio interno condujo a cierta recuperación en el precio de algunos bienes alimenlicios, en especial del frijol y arroz. Sin embargo, tal parece que los aumentos de precios no han beneficiado tanto a los productores como a los que controlan la compra-venta de los alimentos.

Para antes de 1989 , se podía argumentar que si el estado salvadoreno, desde la creación del Instituto Regulador de Abastecimientos, no llegó a comercializar más del $20 \%$ de los volúmenes de producción de granos básicos, entonces es que su incidencia sobre el nivel de precios de los mismos no lue tan grande y su responsabilidad en los bajos niveles de precios no fue tan significaliva. Adicionalmente, se puede hacer el sef́alamiento de que en la política de comercialización existie-

4. Acá limilaremos los análisis a los granos básicos, donde la política de comercialización y precios ha sido más explícita.

5. Esta política de comercialización de alimentos básicos, antes de 1989, estaba a cargo del Instituto Regulador de Abastecimientos (IRA). El grueso de las importaciones de granos básicos y leche en polvo eran llevadas a cabo por este organismo gubernamental. 
ron dos tipos de precios, el pagado a los productores (precios de garanlia) y el que se ofreció a los consumidores; y que si hubieron niveles bajos en los últimos, los niveles de los primeros fueron allos. Por tanto, sólo un $20 \%$ de incidencia en la comercialización de granos, los bajos precios de venta de éstos no se debieron lanto a la política del gobierno, sino a las leyes del mercado, mienlras que los altos precios de garantia si serían resultado de la política gubernamental.

Pero como dice parte del refrán popular, "todo depende del crislal por donde se mira". En primer lugar, la capacidad de incidencia de la política gubernamenlal sobre los niveles de precios de los granos básicos no depende sólo del porcentaje de cobertura de la política de comercialización interna. Más allá de que una cobentura del $20 \%$ de la incidencia (además si la cobertura oficial se ha creado es precisamente para evitar que los precios suban), los niveles de precios pueden ser muy afectados desde otras políticas agrícolas que tienden, en el corlo o mediano plazo, al aumento de la oferta de granos. Es preciso indicar que con esle tin la polílica agraria del eslado salvadorefio parece haberse inclinado más por las políticas agricolas que, como la de imporlaciones, van en contra de los intereses de los produclores de granos; en lugar de impulsar polílicas agrícolas que, como la de crédilo, asistencia técnica, de exlensión de la producción y produclividad, tienden a lavorecer dichos productores.

Lo dicho en el párrafo anterior nos anima a seguir creyendo que la polílica de comercialización conlribuyó al mantenimiento de los bajos precios de los granos básicos, y que su cobertura del $20 \%$, más que expresar su falia de incidencia en los mismos, es un reflejo más de la politica deflacionaria que tienden a promover los sectores urbanos y el tipo de industrialización que se ha seguido en este pais. Si la polílica agrícola de comercialización interna se contentó solamenle con cubrir el $20 \%$ de la producción, es quizá porque los ya bajos niveles de precios de los granos no exigieron más. Cuando los precios comenzaban a subir, la política interna de comercialización no tendió a ampliar sus capacidades internas de conlrol, simplemente recurrió a las importaciones. En ta! sentido, la falta de cobertura de la política de comercialización no fue tanto un problema de falta de recursos como de volunlad política y/o estrategia económica, es decir de un lipo de polílica agraria.

En segundo lugar, tampoco la existencia de un diferencial de precios dentro de la politica de comercialización a los supuestamente altos precios de garantia, son argumentos que evaden las responsabilidades de la política gubernamental en el mantenimiento o promoción de precios disminuidos en los granos básicos. Aún aceplando que los niveles de precios de garantia hayan sido altos quedarian varias cuestiones pen- 
dientes de solventar. Una de ellas pone en duda que los supuestos altos precios de garantia hayan llegado a la mayoría de pequefios productores, lo cual es plausible dada la limitada, débil y muchas veces corrupta red de comercialización gubernamental. Lo que importa tomar en cuenta por tanlo son los precios reales que reciben la mayoria de productores de granos básicos. Y estos no parecen haber sido altos ni mucho menos. Al contrario, dados aquellos rasgos de la red de comercialización oficial y las ya largamenle descritas condiciones de precariedad en que realizan sus ventas la mayoría de minilundistas, los precios reales que reciben éstos por sus productos poseen niveles bajos. Por tanto, aunque los precios de garantía puedan haber sido poco más elevados que los de venta al consumidor, y más allá de las intenciones o declaraciones, la limilada y trágil politica de comercialización y la precariedad de la economía campesina anularon sus posibles eféclos benéticos entre los productores. $^{6}$

En síntesis, anles de 1989 , ni la poca coberlura de la política de comercialización ni los supuestamenle más altos precios de garanlía que contiene ésta, son elemenlos que niegan la política dellacionista aplicada a los precios de los principales productos del minifundio salvadoreño. La privilegiada política de importaciones de granos contribuia a conlirmarla.

Luego de 1989 , la política de comercialización y precios se caracteriza por la liberalización. Su resultado ha sido la relaliva recuperación de los precios de ciertos produclos alimenticios, en particular de arroz y frijol, y la aparente contención en el deterioro de los precios de maíz y sorgo. Asimismo, la política de banda de precios ha significado cierla estabilidad al precio de los granos básicos (en particular para el maiz, maicillo y recientemente para el arroz).

El relativo aumento y mayor estabilidad de los precios de algunos granos básicos, ha sido puesto como causa principal del signilicativo

6. Lo que si cabría preguntarnos en torno a ese tipo de elementos de la política de comercialización y precios es porque el eslado salvadoreño no desarrolló las condiciones socio-económicas para que los pequeños productores de granos básicos recibieran aquí supuestamente mayor precio de garanlía. Ante ello nos asalta la pregunta es que esa falta de desarrollo se debió más a un problema de recurso, u obedeció más a una polílica agraria que tiende a colocar el minifundio productor de bienes alimenticios esenciales al servicios del proceso de acumulación del capital, en especial del urbano? La siluación del campesino salvadoreño y la limitación, debilidad o nocividad de la mayor parte de las pollticas agrlcolas que conciernen a los pequeños productores agropecuarios, son factores que ubican la polltica agraria en el centro de la respuesta. 
incremento de la cosecha de granos ocurrida en 1990/91, y presentado como gran éxilo de la política gubernamental de comercialización y precios. Sin embargo, ciertas informaciones ponen en lela de juicio tal éxilo:

-Los incrementos de producción no son exclusivos de la cosecha 90/ 91. Eslos venian dándose desde la cosecha 87/88.

- Justamente en los productos donde se aplicó primero el mecanismo de la banda de precios, es decir el maiz y el maicillo, tue donde se experimentaron mayores decrementos en los beneficios netos en la cosecha 90/91.

- Lo anterior induce a pensar que los incrementos en área y producción de maiz y maicillo se debió más a las lavorables condiciones climáticas que a los efectos de la política económica.

- La menor rentabilidad de los cullivos de maiz y sorgo en 1990/91 muestra una gran deficiencia de la política de comercialización y precios: es cierto que pudo haber habido cierlo freno al deterioro de los precios de esos productos, pero no se pudo contener el alza creciente de los costos de producción de los mismos. La política económica tavoreció más el aumenlo de precio del crédito y de insumos que el de los bienes.

Por otra parte, hay que tener en cuenta que una política de incrementos de precios no garantiza mejores condiciones de producción y/o beneficios a los produclores direclos. En primer lugar, porque el autoconsumo posee un porcentaje elevado entre los pequeños productores de alimentos, y por ende son menos sensibles a las alzas de precios de los bienes (no asi a la de factores, sobre todo de insumos y tierra). Para el caso, entre los produclores de granos (menos en arroz) con extensiones menores de $10 \mathrm{HA}$. aproximadamente el $70 \%$ de ellos no vende su producción al mercado (Pleitez, et, al., 1991, p. 60). En segundo lugar, es de considerar el hecho que, dada la estructura concentrada del mercado de granos, cerca del $35 \%$ de los posibles benelicios brulos van a parar a manos de los intermediarios (lbid., p. 66). Por tanto, mayores aumentos de precios pueden ser altamente captados por los comercianles y usureros, y dejar poco o nulo margen de beneficio a los productores directos.

En conclusión, ni la polílica de conlrol de comercio y mantenimienlo de bajos precios de los alimentos básicos impulsada antes de 1989, ni la política de liberalización de la comercialización y de recuperación de precios aplicada bajo los PEE/PAE luego de 1989, pueden ser evaluados como factores que estimulan los niveles de suliciencia de la producción nacional de alimentos. Al contrario, la política aperturista y liberal que propugnan los PEE/PAE en cuanto a comercio y precios, puede incluso llegar a atentar contra las disponibilidades alimentarias y la auto- 
nomía del pais. Eslo fundamentalmente a través del dalo que dichas politicas están causando a las trágiles y desprotegidas economias campesinas, al exponerlas premaluramente a una compelencia desleal e injusta (ver anterior nola a pie de página.)

\subsubsection{La polftica fiscal y la politica financlera-crediticla}

\section{a. La polftica flscal}

Una constante de la política tiscal, tanto antes y sobre lodo después de 1989, ha sido la reducción del déficit fiscal sobre la base de la disminuación de los gastos, más que por medio del incremenlo de los ingresos.

La política conlractiva del gasto público ha incidido negativamente sobre la seguridad alimentaria de los salvadorehos. Como bien lo sef́ala la FAO, " existen relaciones directas e indirectas entre el recorte del gasto público y la seguridad alimentaria" (FAO, 1990, pp. 41)

Los efectos directos de la disminución del gaslo público en el sector agropecuario (que de representar el $8 \%$ del gasto total a principios de la década peso a sólo el 3\%) se han dejado sentir en el abastecimiento de insumos, en los servicios de apoyo, en la intraestructura lísica y en los canales de comercialización, entre otros. En primer lugar, la eliminación de subsidios a la compra de insumos ha favorecido el incremento de los costos de producción de los alimentos. En segundo lugar, los menores gastos en investigación y asistencia técnica vienen dafiando el polencial y la productividad en el cullivo de bienes alimenticios. En tercer lugar, la disminución de gastos en intraestructura física limita aún más los escasos equipamientos necesarios a la producción y comercialización de alimenlos; eslo, junto a la merma de los servicios de apoyo, son elementos que favorecen el incremento de las pérdidas posl-cosecha. En cuarto y úllimo lugar, la conlracción del gasto público por medio del cierre o recorte de operaciones de empresas estatales vinculadas al agro (como el IRA), favorece tendencias concentradoras en el comercio privado de alimentos o insumos (aunque la liberalización del comercio exterior empuje a una tendencia contraria, al lavorecer la reducción de los márgenes de comercialización).

A nivel hipotético podria plantearse que los efectos indireclos de la reducción del gasto público sobre las disponibilidades alimentarias se hacen sentir vía faclores como la productividad del trabajo y la demanda polencial de alimentos. Por un lado, la reducción del gasto social (salud y educación sobre todo) atenta contra la productividad de los trabajadores y/o produclores de alimentos (que pueden verse más entermos y menos 
capacilados). Por otro lado, organismos más expueslos a las enlermedades y con menores posibilidades de curación, son organismos que requieren mayores raciones alimenlicias, con lo que la demanda polencial incremenla y con ello la necesidad de disponer de más alimenlos para satislacer adecuadamente las necesidades de la población.

\section{b. La política financlera-crediticla}

En primer lugar, la tendencia (no lineal) a la disminución del crédito, en especial para el sector productor de bienes agropecuarios de consumo interno, experimentada tanto antes como después de 1989, no ha favorecido la producción nacional de alimentos. Las propuestas del gobierno de ARENA de abrir y aumenlar líneas de crédilo para la comercialización interna y exlerna de granos lodavía no se han concretado. De ahi que la politica crediticia, al no estimular la producción nacional y facilitar las imporlaciones, no marcha a favor de potenciar las disponibilidades alimentarias (desde la perspectiva de la suficiencia nacional).

En segundo lugar, el mantenimiento del problema estructural de la concentración del crédito entre los grandes productores, lanlo antes de 1989 como bajo los PEE/PAE, sigue conviertiéndose en obstáculo al desarrollo de la producción de alimenlos y por ende al logro de mejores niveles de suficiencia para la producción nacional de los mismos. En eleclo, la orientación prelerencial de los escasos recursos crediticios hacia la agroexportación (caté sobre lodo), va en detrimenlo de la producción interna de alimentos.

En tercer lugar, duranle la elapa de lasas de inlerés reales negativas y líneas de crédito preferenciales (antes del 89), se dió cierlo subsidio al subsector de alimentos, en esencial de granos básicos, asi como a las actividades del llamado sector relormado. Aunque esto lendió a beneficiar la producción nacional de alimenlos, la falta de acompañamiento de otras políticas y medidas (poca asistencia técnica, talta de mejoras en la comercialización, sobrevaluación del tipo de cambio, concentración del crédilo y/o poco acceso al mismo por parle de los pequeños productores, etc.), anuló gran parte de aquellos posibles beneficios.

Durante la etapa de lasas de interés reales positivas y reducción de líneas de crédilo preferenciales (luego de mediados del 89), prácticamente se fue eliminando el subsidio a los relativamente pocos productores de alimentos que tenian acceso al crédito. Mientras esto sucedia, se continuó, como antes, con una falla de acompañamiento de otras políticas de apoyo al sector. El resultado global de la política financierocrediticia fue entonces de contribución a la pérdida de suficiencia en la producción nacional de alimentos, e incluso de poco eslímulo a la impor- 
tación de los mismos (contrariamente a la política cambiaria y arancelaria), es decir, de afectación de las disponibilidades alimentarias.

En cuarto y último lugar, valga advertir que la política de contención de la inflación de estos úllimos anos, lograda en parte por medio de la política financiero-crediticia, junlo a la posible ampliación del diferencial de precios de los alimentos a nivel regional, puede conducir a promover las exportaciones de alimentos. Esto podía alentar contra las disponibilidades alimentarias nacionales.

\section{Incidenclas de la politica económica sobre otros parámetros de la seguridad allmentarla. Algunos planteamlentos hlpoléllcos}

En esta segunda parte pretendemos avanzar algunos planteamientos hipotéticos en torno a las incidencias de la polílica económica sobre otros de los parámetros en que basamos la seguridad alimentaria. Recordemos algunas advertencias y/o delimitaciones antes hechas:

- Concentramos el estudio en los que podriamos denominar "parámetros cualitativos" más representivos de la SA, es decir, la sustentabilidad y la calidad/consumo apropiado de alimentos.

La falta de investigaciones al respecto justifican en parte tal delimitación.

-El análisis de los efectos de la política económica predominante sobre la SA se realiza acá bajo planteamientos hipotéticos. En tal sentido, esta segunda parte Irata de presentar hipólesis más que resolverlas. Los planteamienlos hipoléticos se avanzan con el fin de que puedan servir como punto de partida de olra investigación, que dé continuidad a este primer esfuerzo investigalivo.

1. Incidenclas de la política económica en la sustentabilidad/viabilidad ecologica en la segurldad alimentaria

En esla sección se prelende, por un lado, establecer las relaciones que exislen entre la sustentabilidad y la seguridad alimentaria. Por otro lado, se busca definir algunos plateamienlos hipotéticos en torno a las incidencias que han tenido determinadas políticas económicas sobre al-

7. La reducción de la tasa de inflación para 1990 y 1991 parece haber sido conseguida en parte por el incremento de las tasas de inlerés, de la reducción de la masa monelaria, del mantenimiento del tipo de cambio sobrevaluado y de la contracción de la demanda de amplios sectores, es decir por medio de mayores niveles de pobreza. 
guna de esas relaciones. Por consiguiente, se contempla analizar los siguientes puntos:

a) Revelar algunas de las relaciones significalivas que hay que considerar dentro de nuestro parámetro de sustentabilidad, en el marco de la SA. Dados los objetivos del presente trabajo, interesa deslacar dos tipos de relaciones: las que se dan entre el ecosistema y la problemática alimentaria, y las que se dan entre las formas de producción en el agro y el ecosistema. La primera relación nos mostrará cómo el deterioro del ecosislema alecta negativamente la siluación alimenlaria. Mientras que la segunda relación permitirá comprender cómo determinadas formas o técnicas de producción agropecuaria (lo que definiremos como modelo tecnológico) favorecen el deterioro del ecosistema. Asi, de la puesla en común de dichas relaciones podemos deducir una tercera relación: ciertas formas de producción o cierto modelo tecnológico aplicados en el agro son faclores que afectan la seguridad alimentaria de los pueblos.

b) Mostrar, en términos hipoléticos, algunas incidencias que han tenido determinadas polílicas económicas (cambiaria, comercial/arancelaria, fiscal, crediticia, de precios) sobre las formas de producción agropecuaria o sobre el modelo tecnológico implementado en el sector agrario. Esto permilirá a su vez acorde a la tercera relación mencionada más arriba, mostrar cómo las políticas económicas han afectado la seguridad alimentaria del país.

\subsection{Algunas relaclones signiflcativas entre sustentabilidad y se- gurldad allmentaria}

Las relaciones entre el ecosislema y la seguridad alimentaria de un país son dialécticas y complejas. Por un lado, los desequilibrios del ecosistema afectan las disponibilidades alimentarias, la accesibilidad y la calidad de los alimenlos, la estabilidad de precios y volúmenes de los bienes alimenticios, etc. Por otro lado, los tipos de cullivo, las formas de producir, almacenar, distribuir, consumir y desechar los alimentos influyen sobre los equilibrios del ecosislema. Hagamos un breve análisis de estos dos lipo de relaciones.

\subsubsection{Ecosistema y segurldad allmentarla}

Diversos diagnóslicos sobre la siluación ecológica de nuestro país coinciden en mostrar el amplio y acelerado deterioro del ecosistema. Todos ellos descatacan la grave situación, todos revelan la alarmante pérdida de bosques, de calidad del suelo, de la cantidad y calidad del 
agua, de la extinción de las especies, etc. Por tanto, no creemos necesario detenernos en una descripción del deterioro ecológico que experimenta EI Salvador. Hemos creido más imporlante analizar las relaciones que existen entre el deterioro del ecosistema y la pérdida de seguridad alimentaria. Esto permitirá comprender mejor cómo la política económica, al alectar los equilibrios de la naturaleza, actúa negativamenle sobre la SA de la nación.

Cierlamente, el deterioro ecológico atenta conlra la SA de los pueblos:

- La pérdida de diversidad genética, vegetal y animal, ${ }^{8}$ producto de gran medida de los desequilibrios del ecosistema, alenta contra las disponibilidades alimenticias y el eslado nutricional de la población. ${ }^{9}$

- La erosión y/o el deterioro de la calidad del suelo, el dafíar la productividad del mismo, alenta contra la capacidad de producir alimentos y de importarlos (porque se dafía lambién los cultivos de exportación y la capacidad de generar divisas).

- Los desequilibrios en la conservación de las especies, distorsionando la selección nalural de las mismas, y por ende potenciando artilicialmente algunas de ellas, lomenta la aparición de plagas que alen-

8. Desde lo que va del presente siglo, del mapa zoológico de El Salvador han desaparecido al menos 13 especies de verlebrados, olras 66 se encuentran en peligro de extinción y 49 confrontan amenaza de extinción. $Y$ para fines de 1970 habían desaparecido cerca de 65 especies de árboles y 61 especies de flores (Véase Dougherty, et. al,. 1979, p. 90).

9. Una consecuencia imporlante sobre la satisfacción de las necesidades básicas y/o la calidad de vida de la mayoría de salvadoreños es la que afecla las posibilidades de enriquecimiento de la dieta alimenticia. Es sabido que hace algunos años buena parte de los habilanles rurales enriqueclan su dieta básica con diversos alimentos, ricos en protelna de alta calidad, que encontraban en la vida silvestre a través de la recolección en árboles Irutales, de la caza de animales y de la pesca esporádica. La extinción o reducción de muchas especies ha dañado gravemente aquellas preciosas posibilidades. No hay duda, por ejemplo, que la drástica reducción de los manglares (bosques marinos importantes para la reproducción de mariscos, aves y reptiles) y otra vegetación costera, asl como el uso masivo de productos químicos en la producción agricola, incidió muy negativamente en la dieta de los campesinos de la costa oriental de país. Esto debido a la casi desaparición de especies, que, como la iguana, garrobo, "cusuco", "cascos de burro", huevos de tortuga, etc., tormaban parte no desdeñable de su dieta. 
tan contra la producción y almacenamiento de los alimentos ${ }^{10}$.

- La contaminación, el uso inadecuado de insumos químicos, los malos mélodos de combale de plagas, la contaminación de aguas superficiales y subterráneas, elc., junto con otros factores que como la pérdida de cubierta vegetal y la erosión, facilitan el "lavado" o circulación de contaminantes, se convierten en elemenlos que atentan contra la calidad de los productos alimenticios.

-El deterioro del ecosistema expone las cosechas de alimentos al riesgo de mayores caláslrofes naturales. Las frecuenles sequias que ha experimentado nuestro país en los últimos afios o los peligros de inundaciones (lo que ocurrió en Filipinas a fines del año pasado es un botón de alarma), son algunos de los problemas que atentan en forma creciente contra la eslabilidad de la oferta anual de bienes alimenticios y de sus precios.

\subsubsection{Formas de producclón, ecosistema y seguridad alimentarla: el rol del Modelo Tecnológlco Especlallzado en los desequi- Ilbrios del ecosistema y en la problemática alimentarla}

\section{a. Dellmilación de las relaclones a estudiar}

La dinámica y rasgos del sislema agropecuario o alimenlario (tipos de cultivo, formas de producir, almacenar, consumir, elc.) delerminan e inciden sobre los equilibrios del ecosistema. Asi, por ejemplo, los cultivos irutales conservan mejor los suelos que el cultivo, formas de producir, almacenar, consumir, etc.) determinan e inciden sobre los equilibrios del ecosistema. Asi, por ejemplo, los cultivos frutales conservan mejor los suelos que el cultivo de cereales, y de la misma forma to hace el cultivo asociado de maíz/trijol que el cullivo de sólo maíz; un patrón de consumo (o un tipo de orientación de mercado) que privilegia en la diela al consumo de carne roja podría tavorecer la delorestación (por la expansión de los paslos ganaderos); y finalmente un modelo o patrón tecnológico basado en el monocultivo y/o uso masivo de insumos quimicos tiende a perturbar el ecosistema.

Por tanlo, la sustentabilidad o viabilidad ecológica dentro de la SA debe considerar tales interrelaciones.

10. El almacenamiento de los granos básicos se ha visio dañado por la modificación de los necesarios equilibrios que deben existir entre las especies. De lal forma que el exterminio de los depredadores naturales de los roedores, $\infty$ mo las culebras, sea uno de los molivos de la mayor y más Irecuente aparición de estos, sobre todo en los lugares cercanos a los centros de almacenamiento de granos. 
En tal senlido, podria lormalizarse nuestro parámetro en los siguientes términos: $s=1(D R N$, IdRN, EE), donde:

$S$ = Sustentabilidad dentro de la SA,

la cual es función de:

DRN = Disponibilidad de recursos nalurales

IdAN = Tasa de depreciación de los recursos nalurales

$\mathrm{EE}=$ Estado de los equilibrios del ecosistema

A su vez, las variables DRN, IdRN y EE dependerian de otras muchas variables que definen un sistema agropecuario o alimentario, tales como TP (tipo de bien cultivado), SC (sistema de cultivo), MT (modelo tecnológico), PC (patrón de consumo), etc. Por tanto, S podrá expresarse también en la siguiente función:

$s=1(T P, S C, M T, P C, \ldots)$

Asi, para conocer los efeclos de una determinada polílica económica sobe $S$ podemos actuar de dos maneras: conociendo direclamente los efectos de la política económica sobre la disponibilidad de recursos, sobre sus grados de delerioro o sobre delerminados equilibrios ecológicos. Otra forma adecuada de esludiar dichos eleclos es analizar las consecuencias que tienen las políticas económicas sobre lactores como TP, SC, PC, MT.

En esle trabajo hemos optado por la segunda forma. En tal sentido, para conocer las incidencias de las políticas económicas en uno de los "parámelros cualitativos" de la SA (la suslentabilidad), lo haremos por medio de su incidencia sobre las variables que se encuentran determinándolo (TP, SC, PC, MT, elc.). Dado el carácler restringido del presente esludio, nos limilaremos al análisis de sólo una de esas variables, es decir, de MT.

Por eso, antes de adentrarnos al estudio de los eleclos de la política económica predominante sobre el parámelro de SA acá considerado, hemos creido conveniente analizar las relaciones que exislen entre el modelo tecnológico que predomina en la agricultura y el estado del ecosistema. Esto permitirá comprender mejor cómo las incidencias de la política económica en la conformación del modelo lecnológico, afeclan la seguridad alimenlaria del país, vía deterioro del ecosistema. 


\section{b. El Modelo Tecnologlco Especlallzado y su rol en los dese- quilibrios ecológlcos y en los problemas allmentarios de EI Sal- vador}

A un nivel general, y retomando las expresiones de Toledo (1990), el Modelo Tecnológico Especializado (MTE) produce la erosión del suelo y la baja de su rentilidad, la salinización y el agolamienlo de las aguas subterráneas en las áreas de riego, la contaminación del medio ambienle a causa de la gran aplicación de insumos químicos, la pérdida de la diversidad genética de las especies cullivadas, la mayor vulnerabilidad a plagas y enfermedades de los monocullivos extensos, y la tendencia a un uso superior de insumos energélicos no renovables, como el pelróleo y el gas natural, y por ende el aumento de los coslos energéticos. Todos estos desequilibrios ecológicos afeclan la capacidad de producción de alimenlos o las mismas disponibilidades alimentarias.

Es más, uno de los pilares menos cuestionados del MTE, como es el uso de los fertilizantes, no parece tan adecuado en si mismo. Eslo en razón de que, como lo anolan Collins y Lappe (1979), eslos produclos químicos que aumentan los rendimientos no contribuyen a conservar o incrementar los componentes orgánicos de la tierra. A la larga, éslo irá alectar los mismos rendimienlos del sistema productivo. Sobre el tema, y con la finura que caracteriza sus análisis, dichos autores advierten: "Depender anles que nada de los lertilizantes quimicos puede conducir a la derrota a largo plazo. Mientras más se depende de ellos y no del estiércol, los abonos orgánicos, la rolación de cultivos y el abono verde, más disminuirá la materia orgánica y las planlas serán menos capaces de absorver el nitrógeno inorgánico de los fertilizanles químicos. Eslo nos ayuda a comprender por qué la agricultura de Estados Unidos, según el biólogo Dr. Barry Commoner, utiliza ahora alrededor de cinco veces más fertilizantes que en 1947 para producir el mismo volumen de cosechas" (Collins y Lappe, p. 150).

La concepción de "modelo tecnológico especializado" la hemos adquirido del excelente trabajo realizado por Toledo (1989) sobre la problemálica ecológica y alimentaria en México. Como afirma este estudio, "lal modelo modifica las condiciones naturales con el objeto de implantar ecosistemas artificiales basados en una sola especie (monocultivos) y sobre superficies extensas, que se mantienen mediante grande insumos energélicos y económicos (maquinaria, fertilizantes, plaguicidas, riego artilicial, etc.)" (Toledo, 1989, p. 22). En referencia al subsector agricola, la investigación mencionada sef́ala algunas condiciones nalurales que son convenientes a la puesta en marcha del MTE: "Existen por lo menos cuatro 'ingredientes' ecológicos esenciales para que el modelo tecnológi- 
co especializado alcance a funcionar dentro de la producción agricola: topogralia plana, suelos fértiles, recursos de agua seguros o muy probables (buen temporal) y ciclos climáticos bien marcados (que atenúen la incidencia de plagas; a ello debe agregarse una superticie lo suficientemente extensa de terreno para que la producción sea rentable..." (Toledo, 1989, p. 36).

EI MTE es buen reflejo de uno de los más grandes males en que basa su reproducción el sistema capitalista: su tendencia a la uniformidad de los produclos, lécnicas, valores, ideas, gustos... e inclusive de la naluraleza. La "homogeneización" de las actividades humanas que lleva a cabo el capitalismo moderno, con su magia (pues esconde la complejidad de la vida y el pensamiento) y su encanto (pues simplifica la vida cotidiana y el "quehacer espiritual! del ser humano), es, como diría Hegel, como la noche, "en la que lodos los galos son pardos". Y ahora que muchos pueblos del llamado Este europeo, hace poco bloqueados por la delormación tolalitaria de sistemas no capitalistas, se dejan seducir por aquella magia y encanto del capitalismo de mercado, creemos pertinenle exponer una de las conclusiones a que arriba el estudio de Toledo: "Existe entonces una contradicción aparentemente insalvable entre la naluraleza misma de la economía de mercado y la diversidad de los ecosistemas" (Toledo, 1989, p. 59).

La aplicación del MTE dentro de los cultivos tradicionales de exportación parece bastante evidente" ${ }^{11}$. Lo es la aplicación de dicho modelo dentro de la producción alimentaria?

Una de las caracteristicas, al tiempo que consecuencia del MTE, como es la especialización productiva, es similar a otro rasgo-efecto de la dinámica agroexporladora, como es la polarización de la estructura agraria. Ambos procesos, de especialización y polarización, conducen a semejantes resultados en el caso salvadoreño: especializar a un polo de explotaciones modernas en los cultivos comerciales de exporlación, y a otro polo mayoritario y no moderno en los cultivos básicos de subsistencia.

En tal sentido, podemos afirmar que el MTE que porta la agroexporlación y que lleva a polarizar la estruclura agraria, se ha extendido al polo productor de granos básicos. Esta expansión puede entenderse de dos maneras. La primera, desde el punlo de vista de la reducción de la diversidad productiva y/o de la imposición del monocultivo al interior del polo de subsislencia. La segunda, desde el punto de

11. Sobre los rasgos y consecuencias de la aplicación del MTE dentro de la agroexportación, consultar Rubio (1991, capítulo 4). 
vista de la imposición de los criterios productivislas/lecnicislas y/o de los llamados "paquetes tecnológicos" dentro del mismo polo.

Aunque no disponemos de estudios e inlormaciones para hacer un análisis consistente sobre la primera lorma de expansión del MTE (y no creemos que hayan muchos), hemos estimado conveniente hacer unos planteamientos al respecto, haciendo conciencia de su nivel teórico o hipotético. Así, una visión panorámica e histórica de los sistemas productivos del campesino salvadorefo nos muestra una evolución tendiente a la reducción de su diversidad productiva. Tal pareciera que buena parte del campesino de anlafio introducía el cultivo de frula y verdura a su sistema productivo basado en granos básicos. Con el tiempo, gran parte de ellos, por razones poco invesligadas, se vieron obligados a especializarse en el cultivo de granos. En fechas más recientes, los cultivos combinados de maíz y irijol y/o maicillo son menos Irecuentes y es más común encontrar unidades campesinas donde sólo se cultiva ef maíz. Con la profundización de la polarización agraria, la agroexportación ha ido introduciendo en el campesinado salvadoreño uno de los pilares del MTE, el monocullivo, destruyendo así "el rasgo que caracteriza ecológicamente a toda economía campesina... la estrategia de uso múlliple" (Toledo, 1989).

Otra forma de expansión del MTE es por medio de la penetración de los criterios y técnicas productivistas en el cultivo de los granos básicos, obviamente entre los medianos y grandes productores de los mismos. Es así que la mini-revolución verde que se aplicó sobre todo en la producción de granos en la década de los setenta, hizo girar los incremenlos de producción en la utilización de semilla mejorada ( $y$ menos en el uso de lentilizantes). Como diria S. Ruíz (1979, p. 80), "el uso de semilla mejorada en los cultivos de granos básicos se difundió en la década de los setenta, llegando a representar en 1975 el $41 \%$ de la superficie cultivada; el cultivo del arroz se realiza en su tolalidad con semilla mejorada; el maiz es cultivado en un $59 \%$ con semilla certificada... En el trijol sólo el $20 \%$ se cultiva con nuevas variedades... y en el caso del maicillo no llega al $8 \% "$.

El monocultivo y utilización intensiva de insumos químicos como criterio fundamental para incrementar la producción que predomina dentro del seclor agroexportador, así como la ampliación de los mismos al sector especializado en la producción de granos básicos, son elementos que muestran la vigencia y dominio de MTE dentro del agro salvadoreno.

A nivel particular, la aplicación del MTE en la agricullura ha generado algunas consecuencias negativas sobre la situación ecológica y 
alimentaria de El Salvador. Mencionemos dos de ellas:

- La implementación del MTE en los cultivos de exportación (en especial en el algodón) ha incidido sobre variables que repercuten desfavorablemente en las capacidades y disponibilidades de la situación alimenticia: suelos menos tértiles, una launa y flora más diezmada, desarrollo de plagas, mayores importaciones de insumos y menores divisas por exportaciones, etc. ${ }^{12}$

-otras consecuencias surgen de la vigencia del MTE dentro de la pesca de exportación (camarón fundamentalmente) y dentro de los productos alimenticios más afectados por la polarización de la estruclura agraria, es decir, los granos básicos.

Ciertamente, el tipo de producción llevado a cabo en la pesca del camarón ha conllevado sus costos ecológicos y alimentarios:

a) La pesca especializada del camarón atenta contra la reproducción a medio plazo de esla especie.

b) La pesca indiscriminada por medio de la técnica de captura masiva en grandes alarrayas, ha arrastrado consigo la muerte de mucha launa de acompanamiento (pescado de escamas sobre todo), entre las cuales se implican muchas especies poco desarrolladas $u$ otras en estado de geslación. Esta pérdida imporlante de la diversidad biológica se añade a la del camarón perturbándose asi seriamente los equilibrios necesarios entre la fauna marina.

c) La especialización se hace acorde a criterios comerciales corloplacistas, por lo que no se toma en cuenta el alto cosio energélico que implica la pesca del camarón: si para obtener una kilocalorías de proteina de anchoveta, peca, bacalao o atún, se necesitaban 2, 4, 20 y 20 kilocalorias de energia fósil respeclivamente, para oblener una de camarón se requerian entre 150 y 206 de energía (Toledo, 1989, p. 51). Todos estos factores mencionados no resultan extraños al hecho de que desde 1964 la pesca del camarón haya disminuido en más de la milad; y que a pesar de dicha baja de producción, enlre 1963 y 1972 el consumo de diesel por unidad de embarcación se duplicó, y el número de naves camaroneras y los días de pesca se incrementaron en un $11 \%$ (AID, et. al., 1985).

En lo que se reliere a los cultivos más afeclados por la "polarización agroexporladora", podemos exponer varias consecuencias negativas. La

12. Para una ampliación de estas consecuencias ver Rubio (1991, capílulo 4). 
tendencia al desarrollo del monocullivo al inlerior de los granos básicos trae aparejado fenómenos semejantes a los presentados en el cultivo de algodón: la disminución de los cullivos combinados de maiz y frijol no contribuye a la fertilidad del suelo, ya que se desaprovecha el intercambio equilibrado de nulrientes que produce la alternancia de ambas plantas (la planla del maiz consume mucho nitrógeno mientras que la del frijol contribuye a fijarlo en el suelo); menos diversidad de plantas significa menos diversidad de insectos y más posibilidad de desarrollo de plagas; además, el monocultivo "ha propiciado la siembra continua de cultivos 'abiertos' como el maíz, que exponen el suelo a la erosión..." (Collins y Lappe, 1979, p. 224).

La utilización de semillas mejoradas que ha impulsado el MTE dentro de la producción de granos básicos no es menos inocente. $Y$ esto no tanlo porque produzca alleraciones perjudiciales al medio ambiente, sino por sus efectos socio-económicos sobre la situación alimentaria. Como lo analizan Collins y Lappe, las semillas mejoradas o las variedades de "alto grado de respuesta" requieren para su aprovechamiento una serie de condiciones que normalmente no están al alcance de los pequeños productores de granos básicos: acceso al agua o dotación de infraestructura para la canalización o drenaje de la misma (dada la mayor sensibilidad de ese tipo de semillas a las inundaciones o sequias), mayores recursos para el control de plagas o entermedades (debido a la menor resistencia a éstas por parte de las semillas mejoradas), acceso a las redes instilucionales que suelen manejar su distribución, mayores conocimientos agronómicos, etc. En consonancia con lo anterior, la difusión de semillas mejoradas entre los productores de granos básicos, en ciertas condiciones, incrementa los costos de producción, aumenta la dependencia y la deuda de muchas explotaciones, arruina a otras, e incrementa las desigualdades socio-económicas entre los productores de granos. Todo esto se traduce de manera desfavorable en la situación alimenticia del país.

Valga advertir, sin embargo, que los problemas ecológicos derivados del tipo de producción que caracieriza los granos básicos, no provienen lanto de la aplicación del MTE y/o de la modernización como de una de sus consecuencias más importantes: la pobreza.

En síntesis, el predominio del MTE en algunos sistemas productivos de los bienes agrícolas o pesqueros de exportación, asi como su aplicación en la producción de granos básicos, son circunstancias que han contribuido a empeorar las precarias situaciones ecológica y alimentaria de El Salvador.

Una vez definidas algunas de las interrelaciones que se dan entre 
ecosistema, lormas o lécnicas de producción agropecuarias y seguridad alimentaria, podemos pasar al estudio de los electos de la política económica predominante sobre el parámetro de la sustentabilidad.

\subsection{Incidenclas de la politica económica sobre la sustenlabilidad dentro de la segurldad allmentarla. Algunas hipólesls de traba- jo}

En este apartado nos limilamos a plantear algunas hipólesis en torno a los electos que, vía afectación del MTE, ha tenido la política económica predominante sobre la sustenlabilidad-seguridad alimentaria en nuestro país. Valga aclarar que, en aras de una mayor aclualización del tema, tomaremos en cuenta solamenle las incidencias de las polílicas económicas pueslas en marcha luego de 1989, es decir bajo el marco del PEE/PAE.

He aquí algunas hipólesis de trabajo al respecto:

-La política cambiaria y comercial/arancelaria, por un lado, al abrir el comercio exlerno y mantener sobrevaluado el tipo de cambio, podría incrementar la utilización de insumos químicos en la agricultura. Este refuerzo de la "quimiquización", sin cambios en los palrones del MTE, tendería a tavorecer el deterioro del ecosistema, tal como ya lo hemos analizado. Esto seria más plausible para la mediana o gran explolación. Pero, por otro lado, la misma apertura del comercio externo, la restrictiva política crediticia, junto con una política de precios que tiende a mantener bajos los precios de algunos bienes alimenlicios y/o altos sus costos de producción, al favorecer la precariedad de los produclores de alimentos y/o granos básicos, podría apoyar la sustitución de semillas mejoradas por semillas criollas (más baratas) ${ }^{13}$.

Esa suslilución de semillas, aunque no sea positiva desde el punto de vista de la rentabilidad, si podría serlo desde el punto de vista de la sustentabilidad. Estas consecuencias pueden ser más senlidas entre los pequeños productores de alimenlos que disponen de excedenles comercializables.

- De la misma forma, las polílicas anles mencionadas, al tomentar la precariedad de los produclores de alimentos, reducen las posibilidades

13. En electo, aunque sólo disponemos de datos de 1971 al 1988 (que señalan que el uso de semilla híbrida de maiz se redujo en un $19.2 \%$ mientras que el uso de la semilla criolla aumentó en $46.4 \%$, tendemos a pensar que se mantiene la lendencia de sustitución de patrón lecnológico, donde las semillas criollas tienden a sustiluir a las semillas mejoradas. 
de mejoras en finca. En un marco de mayor precariedad, los productores no eslán en condiciones de llevar a cabo mejoras en sus explotaciones, sobre todo de aquellas que tienden a preservar el ecosistema (terrazas, cultivos rolalivos, lierras en descanso, siembra de árboles frutales, elc.). Adicionalmente, dadas las relaciones que existen entre el deterioro del ecosistema y la pobreza, esas políticas económicas, al favorecer la precariedad o poca/nula rentabilidad entre los productores de granos básicos (es decir al profundizarse su pobreza), se convierte en un faclor favorable a la degradación ecológica.

- La política de precios impulsada en los dos últimos af́os y tendiente a reaclivar el precio del frijol y arroz, podria estimular el incremento del área cullivada en asocio (frijol y maiz sobre todo). Este incremento puede resultar benelicioso para el mantenimiento de los nutrientes del suelo, y por ende para la preservación del ecosislema. Por otro lado, la polílica de sobrevaluación cambiaria y la comercial/arancelaria, al facililar la importación de frulas y hortalizas, podria deseslimular los sistemas produclivos basados en los cullivos múlliples; éstos, más adecuados para el mantenimiento de los equilibrios ecológicos.

-La polílica fiscal de corte restriclivo, tal como to hacia la política crediticia conlractiva, no es favorable a los esfuerzos de preservación del ecosistema por medio de las mejoras en finca. La reducción de los gastos gubernamentales en salud puede llevar a constreñir aún más los posibles fondos de inversión para mejoras en finca (en la medida que los produclores tendrian que deslinar una mayor parte de sus posibles ahorros a los gastos médicos). La disminución de los gaslos en educación, investigación y asistencia lécnica, dejan a los produclores de alimentos a merced de las necesidades corto placistas e inmedialas, y desprovislos de mecanismos y conocimientos para evitar el deterioro de su ecosislema y/o para aprovechar las polencialidades que los equilibrios de ésle ofrece.

- Las políticas de ajuste encaminadas al fomenlo de la exportación agrícola no tradicional (política crediticia preterencial, política fiscal de exención de algunos impuestos, entre otras), podría representar una mayor expansión del MTE dentro de la agricultura. La entrada al mercado internacional, el apego a sus normas y dinámica, así lo requieren.

\section{La polítlca económica y la problemátlca de la calldad/consumo aproplado de los alimenios}

En esla sección se quiere analizar los efectos que las políticas económicas predominantes han tenido sobre otro de los "parámelros cualilativos" que determinan la seguridad alimentaria de nuestro pais, es 
decir sobre la calidad de los alimenlos y su consumo apropiado. Antes de pasar a plantear las hipólesis sobre dichos efectos, es perlinenle realizar un estudio sobre cómo se presenta la problemática de la calidad y consumo apropiado de alimentos en El Salvador.

\subsection{La problemática de la calidad/consumo apropiado de los ali- mentos: la mala calidad de los blenes alimenticlos ofrecidos y la poca diversidad de la diela de las mayorias.}

A los problemas sobre la situación alimentaria de los pueblos no sólo conciernen las dilicultades que se presenlan a nivel de la suticiencia, la disponibilidad o la accesibilidad de los alimentos, sino que lambién competen las problemálicas referentes a la calidad de dichos bienes y al grado de diversidad de su consumo. Como pretendemos mostrarlo en este punto, la situación alimentaria de El Salvador deja mucho que desear.

\subsubsection{Respecto a la calidad de los blenes alimenticios}

a. La calidad de los alimentos sometidos a un primer proceso de translormación y el uso noclvo de alimentos como materia prima

Antes de entrar en el análisis de la calidad de los productos agropecuarios no manufacturados quisiéramos anadir dos cosas concernientes a la calidad de algunos alimenlos integrados al procesamiento industrial. En primer lugar, sobre la calidad de bienes agrícolas que no son procesados por la industria manufacturera propiamente dicha pero que son resultado de un primer prcesamiento induslrial, como el caso del azúcar producida por la llamada industria azucarera, 0 del pan blanco producido por la industria panadera.

A ese nivel el problema de la calidad de los alimentos tiene fuerte expresión en el caso salvdorefio, noloriamenle en la importante producción azucarera. Esıo por la razón de que el capital agroindustrial, basado en el criterio de la renlabilidad y no en el de la satislacción sana y adecuada de las necesidades humanas, ha escogido un tipo de procesamiento de la caña de azúcar sobre el que parecen pesar serias dudas en lorno a sus consecuencias sobre la salud humana. En efeclo, aunque existe un amplio debate y muchas alirmaciones no tienen más que rango hipotético, se ha deslacado la incidencia del azúcar refinada sobre el cáncer de cólon, a Iravés del eleclo que tiene dicho tipo de azúcar sobre la reducción de fibra dielética. Algo parecido puede decirse sobre la industria del pan blanco y las incidencias en la reducción de fibra dietética y/o aumento de cáncer de cólon. 
He aquí una de las irracionalidades del estilo de desarrollo impuesto por Occidente y adoptado sin reservas en El Salvador: muchos bienes alimenticios se someten a un procesamiento industrial (como el refinado del azúcar y del arroz, o la alla extracción de harina del trigo o de maíz) que da como resultado unos productos más caros, que utilizan más energía en su elaboración y que para colmo no son claramente benéficos para la salud, en lugar de escoger procesamientos más simples, menos cosiosos y más saludables (como el azúcar morena, el arroz y el pan integral). Pero ¿acaso no son estos bienes alimenticios justamente y normalmenle los más caros en el mercado? No nos dejemos caer en el engaño. He aquí una segunda irracionalidad del eslilo de desarrollo predominante: son precisamente los bienes alimenlicios que cuesla menos producir los que se venden más caros (en este caso se entiende), en buena medida debido a la poca demanda que hay sobre ellos... y de aqui otra irracionalidad adicional: se demandan más aquellos bienes alimenticios que pueden ser más perjudiciales para la salud. $Y$ esto no se debe lanlo a un problema de gusto o de "cocina" (el arroz integral tarda más en cocinarse) como de intereses económicos o de "determinaciones culturales interesadas".

Una segunda anotación a hacer apunta a la utilización industrial que se le da a cierlos productos agrícolas. La calidad de los bienes agrícolas como maleria prima puede verse también cuestionada a nivel de sus efeclos sobre la salud humana y/o sobre la calidad misma del bien industrial producido. En lo que compete a la situación salvadoreña podemos traer a cuenta el caso de la ulilización que se hacía del sebo de res en la elaboración de aceites. Acerca del lema, el Instituto de Invesligaciones Económicas de la UCA afirma: "Eslas empresas (se refiere a las industrias de fabricación de acetes y grasas vegetales y animales. $N$. del A.) utilizan como principal materia prima el sebo de res, la cual ha sido prohibida en la producción de grasas y mantecas vegetales por ser dafína para la salud humana; no obstanle esta prohibición, se ha seguido importando, al margen de la resolución del Consejo Ejecutivo del Tratado General de Integración. Resulta incoherente que se olorgue este tipo de exenciones fiscales a las empresas que importen materia prima (sebo de res), no recomendable para la salud" (Instituto de Invesligaciones Económicas, 1985, pp. 113 y 116).

\section{b. La calldad de los blenes agropecuarlos en El Salvador}

La calidad de los bienes agropecuarios puede apeciarse desde diversos aspeclos: su composición química y sus eleclos sobre el funcionamienlo del organismo, su nivel nutrilivo, su sabor o guslo, etc. En nues- 
tra descripción concerniente a la calidad de los alimentos, nos limitaremos a dar algunos dalos en lomo a lo que podriamos definir como "su grado de contaminación".

El alcance del problema de la conlaminación de los alimentos en EI Salvador parece muy amplio. Esto puede ser hipoléticamente comprensible cuando se toman en cuanto los siguientes faclores: el casi nulo control de calidad que existe sobre la producción alimentaria y/o las formas de evadir con cierta facilidad las débiles disposiciones legales existentes: el uso incontrolado y abusivo de sustancias quimicas en la producción agropecuria (como el uso de peslicidas en el algodón, y que llevó en más de una ocasión al rechazo de exporlaciones de productos agropecuarios dirigidos al mercado norteamericano, como sucedió con la suspensión de exportaciones de carne bovina entre marzo y octubre de 1982, o como ocurrió con ciertos quesos provenientes de zonas algodoneras a los que se les descubrió altos contenidos de DDT); las pobres condiciones sanitarias o de higiene que existen en la mayoría de establecimientos encargados de la preparación o venta de alimentos; la escasa infraestruclura para la conservación y/o refrigeración de bienes alimenticios y que cobra mayor relevancia deniro del clima cálido-húmedo del pais; el hecho que los rios y lagos de El Salvador se encuentren entre los más contaminados del mundo; la gran cantidad de agenles transmisibles como las moscas o cucarachas y la talta de voluntad, conocimiento y medios para combatirlos.

Obviamente, no hemos podido tener mucho acceso a la poca inlormación existente sobre el tema. Sin embargo, quisiéramos hacer mención de dos trabajos que hacen referencia al mismo. El primero de ellos, de carácter oficial (AID, et. al., 1985), recoger algunos resultados generales de olras investigaciones; mientras que el segundo, de carácler universitario, investiga parlicularmente la calidad microbiológica de las carnes consumidas en la capilal del país (UCA, 1988). Hagamos una exposición de las citas que parecen de mayor relevancia para la cuestión que nos ocupa:

En el primer trabajo se deslaca:

-"Los eleclos indirectos por contaminación de peslicidas en la salud de los habilantes han sido poco estudiados. Además, la misma situación ha originado la presencia indeseable de eslos compuestos quimicos en alimentos, habiéndose difundido la gravedad del problema por medio de un estudio preliminar realizado en 1974 (Campos, et. al., 1974) sobre compuestos organoclorados en la leche malema".

-"En cuanto a la conlaminación de plaguicidas en hortalizas, se determinó en 1977 que las concentraciones de organoclorados detecla- 
dos, se encuentran dentro de los limiles máximos de residuos permisibles; sobre los plaguicidas fostorados se encontró que el Dipterex, Tamarón y Disysion, sobrepasaban dichos limites".

- Con relación a leche de vaca y productos lácteos, en investigaciones realizadas entre 1976 y 1979, se detectó la presencia de Dieldrín, DDT, Heptacloro Epóxido y Lindano, reportándose valores que sobrepasan los límites permisibles; nolable es el hecho de en la mantequilla, aunque se detecló la presencia de Lindano y DDT, solamenle el $5 \%$ de muestras superaron el límite máximo permisible".

-"según investigaciones realizadas de noviembre de 1978 a marzo de $1979 \ldots$ se enconlró que un $60 \%$ de los aceites y un $78 \%$ de las grasas invesligadas, presentaban residuos de plaguicidas. Cabe destacar que la manteca de cerdo presentaba las mayores concentraciones".

En el segundo vale la pena cilar:

-"Según un estudio realizado en 1981 acerca de las condiciones sanitarias de 72 rastros municipales de la República de El Salvador, el $0.01 \%$ se calificó como bueno, el $23.61 \%$ como regular, el $70.83 \%$ como malo y el $5.55 \%$ como pésimo".

-"Las instalaciones de ambos rastros se encuentran rodeadas de viviendas y tábricas, lo cual es inadecuado. El rastro de Sanla Tecla carece de lugares de enterramiento por lo que depende de recolección de basura para la eliminación de carcasa y animales decomisados. EI Iren de aseo solamenle recoge los desechos hasla que alcanzan una cantidad considerable. Por ello, en el patio proliferan las moscas y larvas, y en los árboles aledaños se conslató la presencia de docenas de aves de carrofía (zopilotes). Todo ello se convierte en fuentes polenciales de contaminación... El piso de ambos rastros mostraba una mezcla de sangre, lodo y contenido intestinal y lue impresionante observar como los empleados arrastraban vísceras y cabezas sobre esta superticie de ambos rastros".

- La carne destinada a mercados se transporta en vehiculos abiertos a la contaminación ambiental y expuesta al sol; la carne destinada a supermercados y carnicerias ocasionalmente se transporta en vehículos cerrados".

-"En general, según los datos, el promedio de carga microbiana de las 4 muestras de mercados fue de 1.090 .000 microorganismos/gramo, el de carnicerías $650.000 \mathrm{~m} .0 / \mathrm{g}$ y el de supermercados lue de 214.433 m.o/g".

Con tales resullados, se puede llegar a la ridicula conclusión (en un país de desnutridos) de que es mejor no comer carne en El Salvador. 
Los problemas presentados a nivel de la carne son una pequeña muesIra de la gravedad de la situación alimentaria, y que se expresa en el hecho de que muchos de los no muy abundantes y poco accesibles alimentos que se encuentran en el pais son un peligro para la salud humana.

\subsubsection{Respecto al tipo de diela que posee la mayoria de los salva- dorefios. El problema de la falta de diversidad en el consumo de alimentos (el consumo Inaproplado)}

\section{a. El problema general de la diversidad en el consumo de los all- mentos}

El grado de diversidad que posee la oferta alimenticia y/o la diela de la población, es un elemento adicional a considerar en una adecuada descripción o evaluación de la siluación alimentaria de un pais. Una oferta o consumo de alimenlos poco diversilicada es un mal síntoma del estado alimentario, en la medida que la "simplicidad en el consumo" es fuente de desequilibrios orgánicos que alectan la salud del ser humano. Puede haber suficiencia y calidad de alimentos, pero si éstos son pocos diversificados, no hay salud y bieneslar. El consumo abundanle o exclusivo de proteinas de origen animal, de las nulrilivas y altamenle valoradas carnes rojas, ha sido ya largamenle denunciado como nocivo para la salud por díferentes nutriólogos y/o inslituciones de salud, tanto nacionales como internacionales (OMS). En un articulo del periódico español "EI País" (Febrero de 1990), algunos historiadores y nutricionistas afirmaban que la verdadera causa de muerte del Rey Enrique VIII de Inglaterra tue el consumo excesivo de carnes y grasas animales, y que muchas de las muertes y enfermedades de la aristocracia inglesa de aquél entonces se debia a su dieta casi exclusiva de carnes y a su desprecio de los produclos de origen vegetal, asociados al consumo de plebeyos y campesinos.

Ciertamente, que no hay término de comparación enlre muertes $y$ enfermedades provocadas por desnutrición y las causadas por mala nutrición y/o sobrealimentación. La carga de irracionalidad e injuslicia que a nivel político, social y económico lleva la primera es muy superior a la segunda. La primera tiene una connotación masiva y generalizada, mientras la segunda concierne más a la exclusividad y elilismo. La primera aparece más como un fenómeno del Tercer Mundo mientras que la segunda como perteneciente a los paises industrializados. Sin embargo, y a pesar de esas opiniones normalmente aceptadas, creemos que el problema de la poca diversidad de la dieta es un problema generalizado que concierne a la salud de los pobres. La "simplicidad de la diela" 
es un problema importante que se affade al prioritario problema de la insuficiencia de esa dieta dentro de los paises del Tercer Mundo. Y si la problemálica alimentaria dentro de los sectores medios y ricos puede ligarse al consumo excesivo de carne, dentro de los pobres puede vincularse al consumo insuficiente y casi exclusivo de granos. Por tanto, al consumo limitado y poco calificado de alimentos de las mayorías, se agrega la problemática del consumo inapropiado (dieta desequilibrada).

De ahí que una polílica alimenlaria bajo un nuevo estilo de desarrollo debe integrar esta problemática, ya que por ejemplo, se podrán llenar los estómagos de los campesinos con abundanle máiz calificado, pero sus organismos seguirán manteniéndose enfermos y mal nutridos (e incluso desnutridos). Las soluciones a las dificultades alimentarias no se reducen "a matar el hambre" sino a "hacer vivir sanamente al hombre" a través del abastecimiento suficiente, accesible, calificado y equilibrado de los alimenlos.

La falta de diversidad de la dieta alimenticia entre los pobres es también una problemática que concierne a los efectos de la modernización y universalización capitalista sobre las masas campesinas y el medio ambiente (el Occidente civilizado no sólo "moderniza" y uniforma los valores, ideas, formas de producción, de organización económica, social y política, sino que también universaliza hasta nuestras papilas guslalivas). En electo, el tipo de desarrollo agrario llevado a cabo por la civilización occidental, con su penetración en las comunidades campesinas, ha favorecido la producción comercial de unos pocos productos en detrimento de la diversidad producliva que existía en las mismas. La destrucción de casi lodos los vestigios de unas culturas indigenas basadas en la producción y consumo diversilicado, como la azteca, maya e inca, no es un fenómeno ajeno a dicho proceso de modernización-universalización.

Esla reducción simplificadora de la situación alimentaria de los productores agrícolas, de sus sistemas productivos y posibilidades de consumo, tiene graves implicaciones sobre su salud y el medio ambiente. Refirámonos a las incidencias de la lalla de diversidad alimentaria sobre la salud. Acerca del tema y analizando el caso mexicano, Toledo (1989, pp. 67-8) sehala que "esludios recientes han demoslrado que existe una estrecha relación entre el proceso de modernización de las áreas rurales y los niveles de nutrición de los productores, es decir, entre desarrollo rural y alimentación. En lo que se considera los estudios más completos y delallados del mundo sobre el tema, Hernández (1974) y Dewey (1982) han mostrado, con base en múltiples datos, mediciones y encuestas, que para los campesinos de una región del Irópico mexicano 
(La Chontalpa, Tabasco), el paso de una esirategia productiva de lipo múltiple y dirigida a la autosubsislencia (de consumo diversificado de alimentos. N. del A.) a una estrategia especializada (agricola o ganadera) de tipo comercial, ha sido la causa principal de su deterioro nulricional... Es muy probable que los 18.4 millones de habilantes de las áreas rurales que se ha registrado sufren de bajos índices de nutrición (véase Chávez 1979), hayan aparecido como resultado de ese proceso".

Sobre el caso de El Salvador no parecen haber estudios detallados sobre el lema. Caben mencionarse algunos intentos de comparación del estado nutricional o de salud de algunas comunidades. Este, a simple vista, se presenta mejor en aquellas comunidades que, como las que habilan algunas islas del Golfo de Fonseca, los alrededores de la laguna El Jocotal o del bosque de Nancuchiname, poseen una diela con imporlanles proleínas de origen marino o disponen de una mayor variedad alimenticia. Sin embargo, las investigaciones no parecen haber tomado cuerpo lodavía y las apreciaciones quedan a nivel hipotético.

\section{b. La pobre diversidad alimentarla de la mayoria de los salvadore- nos}

La composición principal de la diela de los salvadorefios eslá formada por los granos básicos, es decir maiz, frijol, maicillo y arroz: "casi el 100\% del total de familias comen lortillas de maiz y maicillo; el $86 \%$ comen frijoles y un $65 \%$ consumen arroz con cierta Irecuencia" (Ruiz, 1979, p. 93). Esto, evidentemente, no representa ningún problema. Este comienza a plantearse cuando conslalamos que un allo porcentaje de la población hace depender la oblención de proteínas y calorías casi sólo de los granos básicos: "en 1970, el 80 por ciento de la población centroamericana dependia de los cereales y del frijol para satisfacer más del 60 por ciento de su ingesta de calorías y proteinas" (Arroyo y Arias, p. 457). En consecuencia, no seria de extranar que entre los estratos más pobres de la población, más del $90 \%$ de su ingesla de calorias y proteinas dependiera de los granos básicos. Eslos elevados porcenlajes son una de las muestras de la poca diversidad alimentaria de la mayoria de los salvadorefios.

Ahora bien, seria válido pregunlarse ¿hasta qué punto una dieta basada en los granos básicos, en cereales y legumbres es conlraproducente para la salud? La respuesta no es lácil. Ello porque las dietas combinadas de cereales con frijoles son positivas a causa de que las deficiencias proteínicas de los primeros son compensadas por las proteinas de los segundos; las carencias de aminoácidos en el maiz que afeclan la caldad de su proleina, como la lisina y el tripłólano, son en parte llenadas por las 
que conliene el frijol. Como dirian Collins y Lappe, "no es sólo accidental que en México se combine maiz y frijol, arroz y lentejas en la India, arroz y soya en Japón. Estas mezclas producen proleína biológicamente más aprovechable que cuando la dieta se basa en un solo alimento. Cuando ambos productos se ingieren junlos las diterentes pautas de aminoácidos (los ladrillos de proteina) compensan sus mutuas deliciencias" (Collins y Lappe, 1979, p. 141). Es más, el cultivo combinado de maiz y frijol, como lo señalan los mismos aulores, es más adecuado para la nutrición y fertilización natural de los suelos (el máiz consume mucho nitrógeno mientras el cullivo del frijol contribuye a fijarlo en la tierra). Pero como dijimos, la respuesta no es fácil, como tampoco es lácil aceptar que la diela basada sólo en granos básicos sea adecuada para la salud, a pesar del reconocimiento de lo recién dicho. Expongamos a continuación las razones de nuestra falta de convencimiento.

En primer lugar, nos parece que un consumo basado en los granos básicos no representa una incidencia nociva sobre la salud a condición de que se dé en un contexto de mayor diversidad alimenticia. Tómese en cuenta que no todas las deficiencias del maíz se ven compensadas por el frijol y viceversa. El aprovechamienlo biológico de las proteinas puede tener mayores posibilidades en la medida que en el consumo alimenticio se integren olras variedades de granos y legumbres y otros produclos agricolas y pecuarios. Por lanto, en un contexto de poca diversidad alimentaria para los sectores populares dicho aprovechamiento biológico se ve seriamente limilado. Este contexto corresponde al caso salvadoreno $y$ en particular a su medio nural, en donde parece existir un pobre consumo popular de verduras y frulas, carnes, productos lácteos, huevos y olros granos y legumbres.

En segundo lugar, hay que tomar en cuenta que los granos básicos más consumidos por la población, como el frijol y en especial el maiz, aunque ricos en calorias y carbohidralos, son baslante delicienles en proleinas. Por ejemplo, si se comparan las "pupusas" de trijoles (lortillas de maiz rellenas de trijoles) con el queso duro, los resultados, por 100 grs. de porción comestible, serían los siguientes: las pupusas de frijol contendrian 204 calorías y sólo 6.3 gramos de proleína, mientras que el queso dispondria de 355 calorias y casi el cuádruple de proteinas (23.4 gramos) (Merino, 1988, pp. 10-11). En consecuencia, una diela basada en granos básicos, en un contexlo que limila el aprovechamiento biológico de las bajas cantidades de proleina que conlienen sus principales produclos, es una diela que contribuye al funcionamiento de un organismo con déficits importanles de proleínas. Las consecuencias de esle déficit son lan graves como conocidas y aleclan sobre lodo el adecuado luncionamiento de los tejidos, el volumen muscular, la contextura de la 
piel y, lo más delicado, expone seriamente el organismo a las infecciones. Con estas informaciones, no es difícil describir algunos rasgos comunes de los trabajadores rurales de El Salvador: su baja estatura, limitado volumen muscular, arrugamiento y plegamiento de la piel, envejecimiento premaluro, bajas defensas orgánicas y/o allos indices de mortalidad a causa de las que podrían ser ligeras inlecciones.

A propósilo de la presencia de una "diela popular" de bajo contenido proteinico pero de relalivamente alto contenido energético, cabria hacer la siguiente reflexión. El relativamenle allo contenido calórico del maíz y los frijoles, y la existencia destacable de algunos minerales en algunos de eslos productos, contribuyen a potenciar, en términos energéticos, a los trabajadores agrarios. El hierro de algunos lipos de frijoles, por ejemplo, al favorecer la Iransformación del oxígeno, contribuye a evitar la fatiga premalura. Por ello, y sin ánimo de eslablecer una relación simple y mecánica, es válido pregunlarse hasla qué punlo la lógica del estilo de desarrollo predominante en El Salvador ha estado interesada en el mantenimienlo de una diela popular de alto componente energético que liende a lavorecer la productividad e intensidad del trabajo y la "salud económica" de las plantaciones, y no otro tipo de diela más equilibrada que beneficie la salud y el desarrollo cerebral de sus trabajadores. Si en este mundo de intereses "ni las matemáticas son inocentes" ¿porqué han de serlo los frijoles?

Valga afiadir finalmente que el problema de la falta de diversidad en el consumo de alimentos es algo que concierne especialmente al sector rural. En efecto, la diela urbana es más diversificada. El consumo de los preparados alimenticios populares está mucho más exlendido en las ciudades. $Y$ esios preparados, como los chorizos, encurtidos, lamales, nuégados, empanadas, pupusas, horchalas, etc., se componen de una gran variedad de alimenlos, cuyas composiciones químicas resultan más lavorables para una mejor nutrición. Hay que prevenir, sin embargo, que estos productos están expueslos a allos niveles de conlaminación, la calidad de los productos animales no es muy buena (muchas visceras y "desperdicios"), y exislen allos contenidos de grasa. Más allá de razones genéticas, no es casual que entre los hombres, y sobre lodo entre las mujeres urbanas pertenecienles a los seclores populares (como las llamadas "sefioras de los mercados"), se encuentre relativamente presente el problema de la obesidad.

Donde el consumo es muy simplificado es en el campo. Acá la diela diaria se compone esencialmenle de maiz, sal y frijol. El acceso a los llamados preparados alimenticios es mucho menor y muy poco frecuente en los lugares alejados de las ciudades o pueblos. La obtención de 
bienes perecederos, como las verduras, frutas, lácleos o carnes, en caso de no producirlos y de disponer de poder de compra, se ve seriamente obstaculizada por la casi lotal carencia de infraestruclura de comunicación y conservación. Aunque no disponemos de informaciones esladisticas al respecto y muchas de nuestras afirmaciones quedarán a un nivel todavia hipotélico, traigamos a cuenta algunos resultados de la investigación de $\mathrm{S}$. Monles sobre el agro salvadoreño. En este trabajo de campo, de los cuatro municipios encuestados, sólo en uno de ellos, Coatepeque, el aulor descubre cierta diversidad alimentaria. Para el reslo, como en muchos lugares de la campiña salvadorefia, se puede Iraer a cuenta una constatación que se hizo en el municipio de Aguilares y que puede ser aplicable a buena parte de las zonas rurales: "La comida habilual es: 1) Desayuno: frijoles, tortillas y calé de maiz; 2) Almuerzo (en el lugar de (rabajo): lortillas con sal, trijoles y caté (a esta diela fundamental se añaden olros produclos de vez en cuando: sopa de irijoles, o de verdura con hueso de res, huevos, pan dulce, mantequilla $y$, muy raramente, pollo); 3) Cena: Irijoles, tortilla y calé, y algunas veces un pedacito de queso" (Montes, 1986, p. 50).

Expuesta la problemática sobre la calidad/consumo apropiado de los alimentos, pasemos a ver cómo inciden en ella las políticas económicas predominantes.

2. Incidenclas de la politica económica sobre la calldad y el consumo aproplado de allmentos. Algunas hlpótesis de trabajo

- La política de sobrevaluación cambiaria, junto con la política comercial/arancelaria, al facilitar las importaciones podria contribuir a un consumo más diversificado de los alimentos (aunque para que ello sea efectivo habria que resolver los problemas de accesibilidad). Sin embargo, tales políticas podrian también ir en contra de nuestro parámetro.

Por un lado, la apertura comercial a los productos exIranjeros puede significar la importación de ciertos bienes procesados poco apropiados y/ o de menor calidad alimenticia (nos referimos a la importación de lo que podriamos denominar como "bienes alimenticios basura", que responden a demandas de poblaciones sobrealimenladas de los países del Norte, 0 a la importación de bienes que, como el trigo deslinado a la producción de pan blanco, tienden a sustituir bienes nacionales más nutritivos, como la lortilla de maiz). Por olro lado, aquellas políticas económicas, al ir fomentando el deterioro de la ganaderia nacional (tal como lo mencionamos anteriormente), y (rente a las dificullades que existen en la importación de carnes y grasas, podrían traducirse en pérdidas en las posibilidades de ampliar la diversificación de la dieta. Esta situación no es ajena al hecho 
que "en El Salvador se redujo de manera importanle el mpulsada en gran medida a través de la política cambiaria y la comercial/arancelaria, se podría tender a modificar ciertos sistemas de producción o patrones tecnológicos entre medianos y grandes productores, que pueden incidir negativamente sobre la calidad de los productos alimenticios: mayor importación de insumos o sustancias quimicas que pueden aumentar el riesgo de contaminación de los alimentos; importación de ciertos mélodos o sustancias que alteran la composición de los alimenlos (como la aplicación de hormonas u otras sustancias en la producción de carne). Hay que añadir, sin embargo, que las lacilidades a la imporlación pueden lambién contribuir a elevar el nivel de calidad de los productos alimenticios, como por ejemplo con la importación de equipos para relrigeración, conservación, envasado, etc. de los alimentos.

-Al mismo tiempo, mientras las anteriores políticas pueden conducir a la puesta en marcha de mecanismos más exigentes en el control de calidad de los alimenlos (por las exigencias que se desprenden de la apertura comercial y de la necesidad de fomento a las exportaciones), la política fiscal de reducción del gasto en salud, asistencia técnica e investigación, podría ir en sentido inverso. En eleclo, los menores presupueslos gubernamentales dejan poco margen a la mejora de las oficinas de control de calidad, a los guslos de manlimienlo e higiene de mercados y rastros de las municipalidades, a las posibles mejoras en el manejo de calidad en la producción, disıribución y consumo de los bienes alimenlicios por medio de la asistencia técnica o programas de educación y/o fomento, etc.

-Finalmenle, el conjunto de las políticas cambiaria, fiscal, comercial/ arancelaria y de precios, que, como hemos mencionado anteriormente, han conducido y parecen eslar conduciendo al deterioro de las condiciones de producción y vida de muchos pequefos productores, podría inducir a pensar que, con la baja rentabilidad que presentan los granos básicos, los miles de campesinos productores de los mismos tenderian a desplazarse al cultivo de olros productos alimenticios. Con ello se estaria favoreciendo el logro de mayores niveles de diversidad en la producción y el consumo.

Sin embargo, ante tal argumento podemos anteponer olros. En primer lugar, la racionalidad de subsistencia que predomina entre los pequeños productores de alimentos, no favorece cambios importantes en los sistemas de cultivo, aún frente a modificaciones en el entorno macroeconómico. En segundo lugar, las condiciones de pobreza en que se desenvuelven dichos productores limita enormemente las posibilidades de desplazarse a olros cullivos y/o diversificar su producción y consumo alimen- 
licio. Al contrario, ello puede conducir a restringir, simplificar o empobrecer su dieta, como podría estar sucediendo con la creciente suslitución del maicillo por maíz en el consumo del campesinado pobre. En tercero y úllimo lugar, la indiscriminada política gubernamental de desplazar a los productores de granos básicos hacia la exportación de cultivos no tradicionales, más bien podria orientar imporlantes recursos al mercado externo, en lugar de oientarlos a una producción más calilicada y diversificada de alimentos de consumo interno.

\section{Rellexiones flnales}

Un balance general de los efeclos de las polílicas económicas predominanles sobre la seguridad alimentaria de los salvadoreños, tanto antes como después de 1989, arroja un saldo negativo. Ello sin menoscabo de los efectos posilivos deslacados durante la investigación.

A lo largo del trabajo pudimos apreciar como dichas politicas macroeconómicas han contribuido a mayores niveles de insuficiencia en la producción alimenlaria nacional, han afectado o ponen en peligro de afeclar las disponibilidades alimentarias globales, han atentado contra los equilibrios de un ecosistema necesario al sistema alimentario, y han lavorecido un mayor delerioro en la calidad y consumo de los alimentos. En delinitiva, las políticas económicas predominantes han empujado más hacia la inseguridad alimentaria. Las conclusiones a las que hemos llegado ponen al menos en cuestionamiento la conveniencia o viabilidad de las políticas económicas predominantes, en especial las que se impulsan bajo los PEE/PAE. Ahora bien, podrá argumentarse que los efectos nocivos de las políticas económicas que acompañan estos programas es el precio inevitable que hay que pagar por la estabilidad y eficiencia del sislema económico en su conjunto. El argumento no es tácil de rebalir: ¿cómo no eslar de acuerdo con la eslabilidad y la eficiencia? Sin embargo, no hay que caer en los engaños que encierran este tipo de sofismas.

En primer lugar hay que pregunlarse ¿de qué tipo de estabilidad y eficacia se trala? Evidentemente, no es 10 mismo implementar determinadas políticas económicas cuando se "absoluliza" la estabilidad que cuando la relalivizamos. En este caso, las políticas económicas pueden aceptar, en determinadas condiciones y bajo ciertos objetivos algunos desequilibrios macroeconómicos puntuales. Es más, ésios pueden ser en delerminados momenlos adecuados (por ejemplo un déficit liscal coyuntural en aras de superar una crisis social en un período preciso).

En segundo lugar ¿quién asegura que la única forma de estabilizar y promover la eficacia sean las políticas económicas que acompanan los PEE/PAE? ¿Es que toda corrección de los desequilibrios macroeco- 
nómicos tiene necesariamente que pasar por la afeclación de la producción o de la seguridad alimentaria? ¿Es que acaso no hay olras maneras de geslionar los equilibrios macroeconómicos que no compromelan las disponibilidades alimenlarias y la calidad del ecosislema, de los alimentos y del consumo? Creemos que las políticas económicas de los PEE/PAE no lienen vocación universal, y que no poseen el monopolio de la estabilidad y la eficacia. Creemos que pueden corregirse los desequilibrios y gestionarse los equilibrios macroeconómicos, no sólo sin ir en contra de la seguridad alimenlaria, sino potenciándola.

Queremos aprovechar eslos últimos párralos que nos quedan para proponer algunos lineamienlos de política económica que, buscando la eslabilidad y la eficacia, puedan, desde olra perspectiva, contribuir al reforzamiento de la seguridad alimentaria. He aqui, a manera de ejemplos, unos planleamientos al respecto.

-En el marco de la corrección de los desequilibrios internos y de las medidas anti-inflacionarias, las políticas credilicia y fiscal restrictiva no tienen por que traducirse en acciones contra los productores de alimen1os. En lugar de limitar el crédito y los gaslos públicos indiscriminadamente, las restricciones deberian ser seleclivas. Donde habría que limitar es en el uso del crédito especulativo o "fugitivo" (el escándalo y crisis de las "saves and loan" en los Estados Unidos, o para no ir más lejos, los desfalcos bancarios en El Salvador, como el del Banco Agricola, son pruebas del uso y expansión nociva el crédito), en las lacilidades credilicias para la importación de bienes sunluarios y/o bienes no esenciales (llama la atención el fuerte incremento de las importaciones de bienes de consumo duradero, dentro de las que las importaciones de automóviles han llegado a ocupar el segundo lugar), en los gastos militares, en los gastos públicos burocráticos o innecesarios, etc. En tal sentido, el crédito y los gastos, sobre todo sociales, deben y pueden expandirse efectivamente entre los productores de alimentos y acompanarse de la asistencia técnica adecuada.

-Asimismo, los intentos de corregir los déficit liscales no necesariamente tienen que hacerse a costa de la reducción de gastos que apoyan el sistema alimentario. Ello puede buscarse, en adición a olros ingresos, por medio del incremento de unos ingresos fiscales, cuyas cargas puedan ir a favor de la mejor ulilización de los recursos naturales y/o productivos. Es el caso de los impueslos sobre las tierras improductivas, las mullas a las "infracciones al ecosistema", los impuestos suplementarios a las actividades que tiendan a deleriorar el medio ambiente, etc.

-Una política de liberalización de precios y/o recuperación de los 
mismos pierde efectividad si no hay medidas complementarias que ataquen la esiructura monopolista de los mercados (tal como sucede con la comercialización de muchos alimentos en nuestro pais). En este sentido, una polílica de "liberalización" (en el caso hipotético de aceplar su dudosa conveniencia) no debe limitarse a los precios, sino llevarse a las concentradas estructuras del mercado y de la propiedad de los medios de producción. Hay que "liberar" la propiedad de la tierra y de los bancos, concentrada en manos de los grandes propietarios, la cual se encuentra muy "amarrada" a los cultivos de exportación y dejan poco espacio a la producción de alimentos para consumo interno. Hay que "liberar! los mercados del monopolio de los grandes comercializadores y/o acaparadores, para permitir que los productores de alimentos reciban mejores precios por sus productos. Por último, cabría señalar que la política de liberalización de precios, en caso de implementarse, no debería excluir cierto control de los mismos, al menos indirectamente. El mecanismo de la banda de precios es un buen ejemplo de que ello es posible y puede resullar conveniente.

- Una política comerciaVarancelaria de corte aperturista puede contribuir a reforzar la seguridad o la inseguridad alimentaria. Refuerza la inseguridad cuando la politica de tronteras abiertas encuentra a unos productores de alimentos con irágiles estructuras produclivas, carentes de apoyo, es decir, sin condiciones adecuadas para enirentar una competencia externa, normalmente desleal (subsidiada y con allos niveles de productividad). Podría contribuir a la seguridad alimentaria cuando, por ejemplo, la mayor competencia obligue a mejorar la calidad de los productos alimenticios y sus normas de higiene, cuando favorezca la importación de lecnología "verde" o "suave", elc.

-De la misma forma, una polílica comercial/arancelaria, cambiaria y de precios internos, tendiente a reducir los déficil en la balanza comercial externa, puede también contribuir a la seguridad o a la inseguridad alimentaria. Puede conlribuir a la inseguridad si la disminución del déficit, al ser buscada más por la via de la promoción indiscriminada de exporlaciones que por la reducción selectiva de importaciones, estimula la producción de cullivos comerciales de exporlación en detrimento de la producción de alimento para consumo interno. Y podria contribuir a la seguridad alimentaria si el déficit comercial tiende a reducirse más por medio del decremento de ciertas importaciones que por el incremento indiscriminado de las exportaciones (por ejemplo la utilización o mayor aprovechamiento de técnicas locales, que, como el uso de abonos orgánicos, podría favorecer la reducción de las importaciones de insumos quimicos).

-Por úlimo, si la eficiencia se hace traspasar (aunque no necesaria- 
mente pasa sólo por ahí) a través de una política de precios que no distorsione las senales del mercado, es decir, por una política cambiaria que tienda a un tipo de cambio único y realista, y unos precios intemos que expresen las fuerzas del mercado, ello no debe omitir o negar la posibilidad de recurrir a los "precios preferenciales" para los productores de alimentos $\mathrm{y} / \mathrm{o}$ al uso de medidas complementarias o compensatorias de apoyo. En determinadas circunstancias, por ejemplo de allos niveles de devaluación cambiaria, podría ser posible tipos de cambio menos devaluados para la imporlación de cierlos equipos indispensables o convenientes a la producción de alimenlos. De la misma manera, unos precios elevados del dinero, unas tasas reales de interés muy positivas, deberian ser acompanadas por una serie de politicas compensatorias que favorezcan los productores de alimentos.

\section{ANEXOS}

\section{Anexo 1}

Retrospectiva, benelicios netos, superticie, producción, rendimiento, precios y costos sobre el maíz. Periodo 1983/84 - 1990/91

\begin{tabular}{|c|c|c|c|c|c|c|c|}
\hline $\begin{array}{l}\text { Ano } \\
\text { Aorlcola }\end{array}$ & $\begin{array}{l}\text { Superficie } \\
\text { (Mz.) }\end{array}$ & $\begin{array}{l}\text { Producción } \\
\text { (OQ) }\end{array}$ & $\begin{array}{c}\text { Rendimiento } \\
(O O M z)\end{array}$ & $\begin{array}{l}\text { Procio } \\
\text { (/OO) }\end{array}$ & $\begin{array}{l}\text { Ingr. Tot. } \\
(\mathrm{Mz})\end{array}$ & $\begin{array}{c}\text { Costos Tot. } \\
(M z)\end{array}$ & $\begin{array}{c}\text { Benel. Nelo } \\
(/ \mathrm{Mz})\end{array}$ \\
\hline $\begin{array}{l}1983 / 84 \\
1984 / 85 \\
1985 / 86 \\
1986 / 87 \\
1987 / 88 \\
1988 / 89 \\
1989 / 90\end{array}$ & $\begin{array}{l}345,000 \\
347,700 \\
362,100 \\
368,100 \\
398,500 \\
402,800 \\
394,700\end{array}$ & $\begin{array}{r}9,633,000 \\
11,461,500 \\
10,769,200 \\
9,500,000 \\
12,575,900 \\
12,956,200 \\
12,794,300\end{array}$ & $\begin{array}{l}27.90 \\
33.00 \\
29.70 \\
25.80 \\
31.60 \\
32.20 \\
32.40\end{array}$ & $\begin{array}{l}21.47 \\
18.18 \\
22.80 \\
33.65 \\
33.62 \\
39.03 \\
48.18\end{array}$ & $\begin{array}{r}599.01 \\
599.94 \\
679.54 \\
868.17 \\
1.062 .39 \\
1.256 .77 \\
1,561.03\end{array}$ & $\begin{array}{l}1,192.47 \\
1,432.21 \\
1,349.52 \\
1,898.47 \\
1,900.32 \\
1,461.87 \\
1,726.80\end{array}$ & $\begin{array}{l}(583.46) \\
(832.27) \\
(669.98) \\
(1,030.30) \\
(837.93) \\
(205.10) \\
(165.77)\end{array}$ \\
\hline
\end{tabular}

Fuente: Tomado de Pleitez y otros (1991), cuadro 75, p. 198. 


\section{Anexo 2}

Retrospecliva, beneficios nelos, superficie, producción, rendimiento, precios y coslos sobre el maicillo. Período 1983/84 - 1990/91

\begin{tabular}{|c|c|c|c|c|c|c|c|}
\hline $\begin{array}{c}\text { Año } \\
\text { Agricola }\end{array}$ & $\begin{array}{l}\text { Superficie } \\
\text { (Mz.) }\end{array}$ & $\begin{array}{l}\text { Producción } \\
\text { (QQ) }\end{array}$ & $\begin{array}{c}\text { Rendimiento } \\
\text { (OQ/Mz) }\end{array}$ & $\begin{array}{l}\text { Precio } \\
\text { (/QO) }\end{array}$ & $\begin{array}{l}\text { Ingr. Tol. } \\
\text { (/Mz) }\end{array}$ & $\begin{array}{l}\text { Costos Tot. } \\
(/ \mathrm{Mz})\end{array}$ & $\begin{array}{c}\text { Benel. Nelo } \\
(\mathrm{Mz})\end{array}$ \\
\hline $\begin{array}{l}1983 / 84 \\
1984 / 85 \\
1985 / 86 \\
1986 / 87 \\
1997 / 88 \\
1988 / 89 \\
1999 / 90 \\
1990 / 91\end{array}$ & $\begin{array}{l}158,000 \\
166,000 \\
163,400 \\
171,500 \\
178,700 \\
174,200 \\
170,900 \\
184,700\end{array}$ & $\begin{array}{r}2,227,100 \\
3,053,900 \\
2,882,800 \\
3,206,800 \\
564,200 \\
3,332,700 \\
3,249.700 \\
3,491,800\end{array}$ & $\begin{array}{r}14.40 \\
18.40 \\
17.60 \\
18.70 \\
3.20 \\
19.10 \\
19.00 \\
18.90\end{array}$ & $\begin{array}{l}18.30 \\
15.39 \\
22.24 \\
29.12 \\
36.90 \\
36.55 \\
43.70 \\
52.57\end{array}$ & $\begin{array}{l}263.52 \\
283.18 \\
391.42 \\
544.54 \\
118.08 \\
698.11 \\
830.30 \\
993.57\end{array}$ & $\begin{array}{c}1,143.69 \\
1,233.46 \\
1,304.37 \\
1,742.29 \\
\text { n.d. } \\
1,196.43 \\
1,224.80 \\
1,439.09\end{array}$ & $\begin{array}{c}(880.17) \\
(950.28) \\
(912.25) \\
(1,197.75) \\
\quad \text { n.d. } \\
(498.32) \\
(394.50) \\
(445.52)\end{array}$ \\
\hline
\end{tabular}

Fuente: Ibid., cuadro 76, p. 199.

\section{Anexo 3}

Retrospecliva, benelicios netos, superticie, producción, rendimiento, precios y costos sobre el frijol. Periodo 1983/84 - 1990/91

\begin{tabular}{|c|c|c|c|c|c|c|c|}
\hline $\begin{array}{c}\text { Año } \\
\text { Agricola }\end{array}$ & $\begin{array}{c}\text { Superficie } \\
\text { (Mz.) }\end{array}$ & $\begin{array}{l}\text { Producción } \\
\text { (OQ) }\end{array}$ & $\begin{array}{c}\text { Rendimiento } \\
\text { (OO/Mz) }\end{array}$ & $\begin{array}{l}\text { Precio } \\
(/ O O)\end{array}$ & $\begin{array}{l}\text { Ingr. Tol. } \\
(\mathrm{Mz})\end{array}$ & $\begin{array}{c}\text { Cosios Tol. } \\
(/ \mathrm{Mz})\end{array}$ & $\begin{array}{c}\text { Benel. Neto } \\
\text { ( } / \mathrm{Mz})\end{array}$ \\
\hline $1983 / 84$ & 80,500 & 918,300 & 11.41 & 48.25 & 550.53 & $1,317.76$ & (767.23) \\
\hline $1984 / 85$ & 82,500 & 1.056 .000 & 12.80 & 55.61 & 711.81 & $1,002.62$ & $(290.81)$ \\
\hline $1985 / 86$ & 83,300 & 761.200 & 9.02 & 75.26 & 678.85 & $1,031.61$ & (352.76) \\
\hline $1986 / 87$ & 87,100 & $1,093,900$ & 12.56 & 97.94 & $1,228.87$ & $1,445.46$ & (216.59) \\
\hline $1987 / 98$ & 69,300 & 531,000 & 5.95 & 148.34 & 882.62 & $1,335.82$ & $(453.20)$ \\
\hline $1989 / 89$ & 96,100 & $1,240,000$ & 12.90 & 160.99 & $2,076.77$ & $1,902.67$ & 174.10 \\
\hline $1989 / 90$ & 91,600 & 968,000 & 10.60 & 180.14 & $1,909.48$ & $1,727.83$ & 131.65 \\
\hline $1990 / 91$ & 89,500 & $1,145,400$ & 12.80 & 234.19 & $2,997.19$ & 2.164 .59 & 033.04 \\
\hline
\end{tabular}

Fuente: bid, cuadro 77, p. 200.

\section{Anexo 4}

Retrospectiva, beneficios netos, superficie, producción, rendimienlo, precios y coslos sobre el arroz. Periodo 1983/84 - 1990/91

\begin{tabular}{|c|c|c|c|c|c|c|c|}
\hline $\begin{array}{c}\text { Ano } \\
\text { Agricola }\end{array}$ & $\begin{array}{c}\text { Superlicie } \\
\text { (Mz.) }\end{array}$ & $\begin{array}{l}\text { Producción } \\
\text { (OO) }\end{array}$ & $\begin{array}{c}\text { Rendimiento } \\
(\mathrm{OO} / \mathrm{Mz})\end{array}$ & $\begin{array}{l}\text { Precio } \\
\text { (/OQ) }\end{array}$ & $\begin{array}{l}\text { Ingr. Tot. } \\
(/ \mathrm{Mz})\end{array}$ & $\begin{array}{c}\text { Cosios Tol. } \\
(/ \mathrm{Mz})\end{array}$ & $\begin{array}{c}\text { Benel. Nelo } \\
(/ \mathrm{Mz})\end{array}$ \\
\hline $\begin{array}{l}1983 / 84 \\
1984 / 85 \\
1985 / 86 \\
1986 / 87 \\
1987 / 88 \\
1988 / 89 \\
1989 / 90 \\
1990 / 91\end{array}$ & $\begin{array}{l}18,000 \\
21,900 \\
24,700 \\
17,200 \\
16.700 \\
19,700 \\
22.200 \\
20.400\end{array}$ & $\begin{array}{r}940,000 \\
1,376,900 \\
1,497,600 \\
1,020,400 \\
914,550 \\
1,245,900 \\
1,385,200 \\
1,341,100\end{array}$ & $\begin{array}{l}55.22 \\
62.87 \\
60.63 \\
59.33 \\
54.76 \\
63.24 \\
62.40 \\
65.70\end{array}$ & $\begin{array}{l}30.66 \\
20.00 \\
24.07 \\
33.60 \\
69.52 \\
53.41 \\
46.25 \\
72.46\end{array}$ & $\begin{array}{l}1,601.07 \\
1,257.40 \\
1,459.36 \\
1,993.49 \\
4.124 .62 \\
3,377.65 \\
2,886.00 \\
4,760.62\end{array}$ & $\begin{array}{l}1,639.73 \\
1,587.53 \\
1,684.03 \\
2,844.31 \\
2,632.43 \\
3.079 .77 \\
3.505 .74 \\
4.025 .11\end{array}$ & $\begin{array}{r}(38.66) \\
(330.13) \\
(224.67) \\
(850.82) \\
1,280.31 \\
297.88 \\
(619.74) \\
735.51\end{array}$ \\
\hline
\end{tabular}

- Promedio simple, incremento de precio debido a espectalivas generadas por la sequla.

Fuente: /bid., cuadro 78, p. 201. 


\section{REFERENCIAS BIBLIOGRAFICAS}

Agencia para el Desarrollo Internacional (AID) y olros (1985), "Perlil ambiental de El Salvador". U.S.A.I.D. Contralo N 519-0167-C-00-2039-00. San Salvador.

Arroyo, Gonzalo y Arias Salvador (1987), "Lineamientos estratégicos para un desarrollo endógeno centroamericano con seguridad y autosuficiencia alimentaria. La contribución de la biotecnología". ECA, № 465 (Julio).

CEPAL-CADESCA (1991), "Istmo Centroamericano: Seguridad Alimentaria y Politica Macroeconómica en el decenio de 1989". Colección temas de Seguridad Alimentaria Ne 9 (Junio).

Collins, Joseph y Moore Lappe Frances (1986), Comer es primero. Más allá del miro la escasez. México: Siglo XXI.

Cruz Letona, Ricardo (1991), "La polltica de granos básicos en El Salvador: Análisis y recomendaciones". Cuadernos de Investigación No 5.

Dougherty, H.E., Jeannerel, C.A. y Fletcher, H.F. (1979), Stratégies d'ecodeveloppement pour le Salvador. Ginebra.

Instituto de Invesligaciones Económicas (1985), "La eslructura de la Industria Alimenticia y las Necesidades Básicas". Boletín de Ciencias Económicas y Sociales (Abril-Mayo), UCA, San Salvador.

Merino, José Gerardo (1988), Composición qulmica de alimentos poulares de EI Salvador". Boletín de Ciencias Naturales y Agrarias № 3 (Abril), UCA, San Salvador.

Montes, Segundo (1986), El agro salvadoreño 1973-1980. San Salvador: UCA Editores.

Organización de las Naciones Unidas para la Agricultura y la Alimentación (FAO) (1990), Estudio FAO Desarrollo Económico Social, N² 89. Roma.

Pleitez, Rafael y otros (1991), Implicaciones de los Programas de Ajuste Estructural sobre los pequeños productores de granos básicos. Tesis de Grado, Universidad Centroamericana (UCA), San Salvador.

Pleitez, William (1991), "Hacia una estrategia integral de seguridad alimentaria en EI Salvador". Documenlo de trabajo CADESCA-PSA, San Salvador.

Rivera Campos, Roberto (1988), "La inflación en El Salvador", Revista Realidad Económico-Social Ne 1.

Rubio, Roberto (1991), El rol de la agroexportación en la conformación de la sítuación alimentaria y ecológica de El Salvador 1950-1979. Louvain-la-Neuve. Bélgica. Tesis doctoral.

Ruiz Granadino, Santiago (1979), "Modernización agrícola en El Salvador", ECA.

Toledo, Viclor Manuel y otros (1989), Ecologla y autosuficiencia alimentaria, México: Siglo XXI.

Universidad Centroamericana (UCA) (1988), "Aspectos de la calidad microbiológica de las carnes consumidas en San Salvador y alrededores". Trabajo elaborado por estudiantes de la cáledra de Microbiología, Boletín de Ciencias Naturales y Agrarias, N², UCA, San Salvador. 